\title{
CONTEMPORANEOUS VLBA 5 GHz OBSERVATIONS OF LARGE AREA TELESCOPE DETECTED BLAZARS
}

\author{
J. D. Linford ${ }^{1}$, G. B. TAylor ${ }^{1}$, R. W. Romani ${ }^{2}$, J. F. Helmboldt ${ }^{3}$, A. C. S. Readhead ${ }^{4}$, R. Reeves ${ }^{4}$, And J. L. Richards ${ }^{4}$ \\ ${ }^{1}$ Department of Physics and Astronomy, University of New Mexico, MSC07 4220, Albuquerque, NM 87131-0001, USA \\ ${ }^{2}$ Department of Physics, Stanford University, Stanford, CA 94305, USA \\ ${ }^{3}$ Naval Research Laboratory, Code 7213, 4555 Overlook Avenue SW, Washington, DC 20375, USA \\ ${ }^{4}$ Astronomy Department, California Institute of Technology, Mail Code 247-17, 1200 East California Boulevard, Pasadena, CA 91125, USA \\ Received 2011 September 19; accepted 2011 November 17; published 2011 December 22
}

\begin{abstract}
The radio properties of blazars detected by the Large Area Telescope (LAT) on board the Fermi Gamma-ray Space Telescope have been observed contemporaneously by the Very Long Baseline Array (VLBA). In total, 232 sources were observed with the VLBA. Ninety sources that were previously observed as part of the VLBA Imaging and Polarimetry Survey (VIPS) have been included in the sample, as well as 142 sources not found in VIPS. This very large, $5 \mathrm{GHz}$ flux-limited sample of active galactic nuclei (AGNs) provides insights into the mechanism that produces strong $\gamma$-ray emission. In particular, we see that $\gamma$-ray emission is related to strong, uniform magnetic fields in the cores of the host AGN. Included in this sample are non-blazar AGNs such as 3C84, M82, and NGC 6251. For the blazars, the total VLBA radio flux density at $5 \mathrm{GHz}$ correlates strongly with $\gamma$-ray flux. The LAT BL Lac objects tend to be similar to the non-LAT BL Lac objects, but the LAT flat-spectrum radio quasars (FSRQs) are significantly different from the non-LAT FSRQs. Strong core polarization is significantly more common among the LAT sources, and core fractional polarization appears to increase during LAT detection.
\end{abstract}

Key words: galaxies: active - galaxies: jets - galaxies: nuclei - gamma rays: galaxies - radio continuum: galaxies

\section{INTRODUCTION}

The Large Area Telescope (LAT; Atwood et al. 2009) on board the Fermi Gamma-ray Space Telescope is a wide-field telescope covering the energy range from about $20 \mathrm{MeV}$ to more than $300 \mathrm{GeV}$. It has been scanning the entire $\gamma$-ray sky once every three hours since 2008 July. The LAT first-year catalog (1FGL; Abdo et al. 2010a) has 1451 sources. The majority of these $\gamma$-ray bright sources that have been identified with radio sources are associated with blazars (685 of 1451). These blazars typically are strong, compact radio sources which exhibit flat radio spectra, rapid variability, compact cores with one-sided parsec-scale jets, and superluminal motion in the jets (Marscher 2006).

Several very long baseline interferometry (VLBI) programs, such as the Monitoring Of Jets in AGN with VLBA Experiments (MOJAVE; Lister et al. 2009b, 2011; Homan et al. 2009) observing at $15 \mathrm{GHz}$, the Boston University program observing at 22 and $43 \mathrm{GHz}$ (Marscher et al. 2010; Jorstad et al. 2010), and TANAMI (Ojha et al. 2010a) observing at 8.4 and $22 \mathrm{GHz}$, along with the LAT collaboration themselves (Abdo et al. 2011) are in place to monitor the radio jets from the brightest blazars such as 3C273, BL Lacertae object, etc. Our sample has a flux limit roughly an order of magnitude below the MOJAVE survey and so allows us to probe the extensions of the radio core/ $\gamma$-ray properties down to a fainter population. Many different radio- $\gamma$-ray correlations have been suggested (Taylor et al. 2007; Abdo et al. 2009a; Lister et al. 2009b; Kovalev et al. 2009; Giroletti et al. 2010; Ojha et al. 2010b; Linford et al. 2011). In our last paper (Linford et al. 2011), we found only a marginal correlation between $\gamma$-ray flux and radio flux density, and only for the flat-spectrum radio quasars (FSRQs). With our new, more contemporaneous and larger sample, we find a strong correlation between the two. By examining a larger sample (1248 objects), we attempt to obtain more definitive insight into several other properties of $\gamma$-ray bright blazars.

In Section 2 we define our sample. In Section 3 we briefly describe the data reduction process for our Very Long Baseline
Array (VLBA) observations. In Section 4 we present data on the $\gamma$-ray flux and radio flux densities of the LAT sources. In Section 5 we discuss differences between sources observed in two epochs (prior to or during 2006 and in 2009-2010). In Section 6, we compare several parameters of the LAT and non-LAT sources. Notes on some individual objects of interest are presented in Section 7 and in Section 8 we discuss our results. Throughout this paper we assume $H_{0}=71 \mathrm{~km} \mathrm{~s}^{-1} \mathrm{Mpc}^{-1}$ and $\Lambda \mathrm{CDM}$ cosmology (e.g., Hinshaw et al. 2009).

\section{SAMPLE DEFINITION}

We obtained time on the VLBA to observe LAT-detected sources from 2009 November to 2010 July. We had a total of seven observing runs and collected $5 \mathrm{GHz}$ data on 232 sources. The first three observing runs were follow-up observations on 90 sources in the VLBA Imaging and Polarimetry Survey (VIPS; Helmboldt et al. 2007) and new $5 \mathrm{GHz}$ observations of 7 sources in the MOJAVE sample. The remaining 135 sources were selected from the Fermi 1FGL as sources which were associated with a source in the Combined Radio All-Sky Targeted Eight GHz Survey (CRATES; Healey et al. 2007) with high $(\geqslant 80 \%)$ probability, had a flux density of at least $30 \mathrm{mJy}$ in CRATES, and were not in VIPS or MOJAVE. Of the 232 sources, 95 are BL Lac objects, 107 are FSRQs, and 30 are other types of active galactic nuclei (AGNs; radio galaxies, AGN of unknown type, and 1 starburst galaxy). Any object that is not a BL Lac object or FSRQ we classify as "AGN/Other." The optical classifications are taken from the LAT First Catalog of AGNs (1LAC; Abdo et al. 2010b). See Table 1 for a summary of our data.

We should note that several of the 1FGL sources are associated with multiple AGNs in the 1LAC. Not all of the associated sources are in CRATES, and not all of them are brighter than $30 \mathrm{mJy}$ at $8 \mathrm{GHz}$. We make a note of those objects with multiple associations in Table 1 . We observed two radio sources for three of the LAT sources in our sample. The LAT source 1FGL J1225.8+4336 is associated with the 


\begin{tabular}{|c|c|c|c|c|c|c|c|c|c|c|c|c|c|}
\hline Source Name & 1FGL Name & Alt. Name & R.A. & Decl. & $\begin{array}{l}\text { Opt. } \\
\text { Type }\end{array}$ & Class & $z$ & $S_{5}$ & $\begin{array}{l}\text { LAT } \\
\text { Flux }\end{array}$ & $\begin{array}{c}\Delta \text { LAT } \\
\text { Flux }\end{array}$ & Core $T_{B}$ & $\begin{array}{c}\text { Open. } \\
\text { Ang. }\end{array}$ & $\Delta$ P.A. \\
\hline F00057+3815 & 1FGL J0005.7+3815 & B2 $0003+38 \mathrm{~A}$ & 00:05:57.180 & $38: 20: 15.18$ & bzq & LJET & 0.229 & 475 & 38.61 & 9.33 & $3.59 \mathrm{e}+10$ & $\cdots$ & $\cdots$ \\
\hline F00193+2017 & 1FGL J0019.3+2017 & PKS 0017+200 & $00: 19: 37.850$ & $20: 21: 45.61$ & bzb & SJET & & 680 & 18.93 & 7.63 & $7.18 \mathrm{e}+10$ & $\ldots$ & . \\
\hline F00230+4453 & 1FGL J0023.0+4453 & В3 0020+446 & $00: 23: 35.441$ & $44: 56: 35.81$ & bzq & LJET & 1.062 & 120 & 45.43 & 20.21 & $2.53 e+10$ & 29 & 9 \\
\hline F00419+2318 & 1FGL J0041.9+2318 & PKS 0039+230 & 00:42:04.550 & $23: 20: 01.21$ & bzq & SJET & 1.426 & 676 & 27.31 & 11.19 & $2.18 \mathrm{e}+10$ & $\ldots$ & $\ldots$ \\
\hline F00580+3314 & 1FGL J0058.0+3314 & CRATES J0058+3311 & 00:58:32.071 & $33: 11: 17.20$ & bzb & PS & 1.371 & 151 & 42.25 & 17.77 & $2.68 \mathrm{e}+11$ & $\ldots$ & $\ldots$ \\
\hline F01022+4223 & 1FGL J0102.2+4223 & CRATES J0102+4214 & 01:02:27.151 & $42: 14: 19.00$ & bzq & PS & 0.874 & 163 & 40.98 & 9.25 & $4.04 \mathrm{e}+11$ & $\ldots$ & $\ldots$ \\
\hline F01090+1816 & 1FGL J0109.0+1816 & CRATES J0109+1816 & 01:09:08.179 & $18: 16: 07.50$ & bzb & LJET & 0.145 & 55.6 & 17.20 & 7.19 & $1.62 \mathrm{e}+10$ & $\ldots$ & $\ldots$ \\
\hline F01120+2247 & 1FGL J0112.0+2247 & CGRaBS J0112+2244 & 01:12:05.820 & $22: 44: 38.80$ & bzb & SJET & 0.265 & 327 & 67.57 & 7.96 & $5.29 \mathrm{e}+10$ & $\ldots$ & $\cdots$ \\
\hline F01129+3207 & 1FGL J0112.9+3207 & $4 C+31.03$ & 01:12:50.330 & $32: 08: 17.59$ & bzq & SJET & 0.603 & 405 & 139.77 & 9.04 & $4.14 \mathrm{e}+10$ & $\ldots$ & $\ldots$ \\
\hline F01138+4945 & 1FGL J0113.8+4945 & CGRaBS J0113+4948 & 01:13:27.010 & $49: 48: 24.08$ & bzq & LJET & 0.389 & 510 & 37.30 & 15.93 & $3.49 \mathrm{e}+10$ & 7 & 3 \\
\hline F01144+1327 & 1FGL J0114.4+1327a & CRATES J0113+1324 & 01:13:54.511 & $13: 24: 52.49$ & bzb & LJET & 0.685 & 106 & 39.15 & 7.82 & $3.82 \mathrm{e}+9$ & 20 & 1 \\
\hline F01370+4751 & 1FGL J0137.0+4751 & OC 457 & 01:36:58.591 & 47:51:29.09 & bzq & SJET & 0.859 & 3150 & 193.96 & 10.34 & $2.61 \mathrm{e}+11$ & $\ldots$ & $\ldots$ \\
\hline F01446+2703 & 1FGL J0144.6+2703 & CRATES J0144+2705 & 01:44:33.559 & 27:05:03.08 & bzb & LJET & $\ldots$ & 305 & 40.30 & 8.50 & $4.75 e+10$ & 24 & -16 \\
\hline F02035+7234 & 1FGL J0203.5+7234 & CGRaBS J0203+7232 & 02:03:33.389 & 72:32:53.70 & bzb & LJET & $\ldots$ & 519 & 63.95 & 28.17 & $8.35 \mathrm{e}+10$ & $\ldots$ & $\ldots$ \\
\hline F02045+1516 & 1FGL J0204.5+1516 & $4 C+15.05$ & 02:04:50.410 & 15:14:11.00 & agn & LJET & 0.405 & 1500 & 29.98 & 12.42 & $9.52 \mathrm{e}+10$ & $\ldots$ & $\ldots$ \\
\hline F02053+3217 & 1FGL J0205.3+3217 & B2 $0202+31$ & 02:05:04.930 & $32: 12: 30.10$ & bzq & LJET & 1.466 & 2120 & 45.21 & 19.21 & $1.25 \mathrm{e}+12$ & $\ldots$ & $\ldots$ \\
\hline F02112+1049 & 1FGL J0211.2+1049 & CGRaBS J0211+1051 & 02:11:13.181 & 10:51:34.81 & bzb & SJET & $\ldots$ & 1060 & 41.30 & 8.45 & $1.61 \mathrm{e}+11$ & $\ldots$ & $\ldots$ \\
\hline F02178+7353 & 1FGL J0217.8+7353 & 1ES $0212+735$ & 02:17:30.821 & 73:49:32.59 & bzq & LJET & 2.367 & 3950 & 77.11 & 34.06 & $1.28 \mathrm{e}+11$ & $\ldots$ & $\ldots$ \\
\hline F02210+3555 & 1FGL J0221.0+3555 & B2 0218+35 & 02:21:05.470 & $35: 56: 13.70$ & bzq & CPLX & 0.944 & 835 & 125.80 & 9.39 & $3.40 \mathrm{e}+9$ & $\ldots$ & $\ldots$ \\
\hline F02308+4031 & 1FGL J0230.8+4031 & B3 $0227+403$ & 02:30:45.710 & 40:32:53.09 & bzq & SJET & 1.019 & 501 & 58.74 & 26.55 & $2.71 \mathrm{e}+10$ & $\ldots$ & $\ldots$ \\
\hline F02379+2848 & 1FGL J0237.9+2848 & $4 \mathrm{C}+28.07$ & 02:37:52.411 & 28:48:09.00 & bzq & LJET & 1.213 & 2420 & 135.56 & 9.70 & $1.50 \mathrm{e}+11$ & 27 & 3 \\
\hline F02386+1637 & 1FGL J0238.6+1637 & PKS 0235+164 & 02:38:38.930 & $16: 36: 59.29$ & bzb & PS & 0.94 & 919 & 427.15 & 12.63 & $1.33 \mathrm{e}+11$ & $\ldots$ & $\ldots$ \\
\hline F02435+7116 & 1FGL J0243.5+7116 & CRATES J0243+7120 & 02:43:30.890 & $71: 20: 17.88$ & bzb & SJET & $\ldots$ & 166 & 25.10 & 8.78 & $9.43 e+9$ & $\cdots$ & $\ldots$ \\
\hline F02454+2413 & 1FGL J0245.4+2413 & B2 $0242+23$ & $02: 45: 16.860$ & $24: 05: 35.20$ & bzq & LJET & 2.243 & 251 & 41.30 & 17.43 & $2.75 e+9$ & $\ldots$ & $\ldots$ \\
\hline F02580+2033 & 1FGL J0258.0+2033 & CRATES J0258+2030 & 02:58:07.310 & 20:30:01.58 & bzb & LJET & $\ldots$ & 66.3 & 18.92 & 7.78 & $1.07 \mathrm{e}+10$ & 35 & -10 \\
\hline F03106+3812 & 1FGL J0310.6+3812 & B3 $0307+380$ & 03:10:49.879 & 38:14:53.81 & bzq & PS & 0.816 & 375 & 41.19 & 18.21 & $2.57 \mathrm{e}+11$ & $\ldots$ & $\ldots$ \\
\hline F03197+4130 & 1FGL J0319.7+4130 & NGC 1275 & 03:19:48.161 & $41: 30: 42.12$ & agn & LJET & 0.018 & 16200 & 213.59 & 10.69 & $1.23 \mathrm{e}+11$ & $\ldots$ & $\ldots$ \\
\hline F03250+3403 & 1FGL J0325.0+3403 & B2 $0321+33 B$ & 03:24:41.160 & $34: 10: 45.80$ & agn & LJET & 0.061 & 357 & 56.29 & 23.97 & $2.15 e+10$ & $\ldots$ & $\ldots$ \\
\hline F03259+2219 & 1FGL J0325.9+2219 & CGRaBS J0325+2224 & 03:25:36.809 & $22: 24: 00.40$ & bzq & SJET & 2.066 & 889 & 58.65 & 24.82 & $1.37 \mathrm{e}+11$ & $\ldots$ & $\ldots$ \\
\hline F03546+8009 & 1FGL J0354.6+8009 & CRATES J0354+8009 & 03:54:46.130 & 80:09:28.80 & agu & LJET & $\ldots$ & 280 & 46.05 & 8.82 & $5.05 e+10$ & 35 & -12 \\
\hline F04335+2905 & 1FGL J0433.5+2905 & CGRaBS J0433+2905 & 04:33:37.829 & 29:05:55.50 & bzb & SJET & $\ldots$ & 240 & 58.78 & 19.56 & $4.17 e+10$ & $\ldots$ & $\ldots$ \\
\hline F04335+3230 & 1FGL J0433.5+3230 & CRATES J0433+3237 & 04:33:40.690 & $32: 37: 12.00$ & bzq & SJET & 2.011 & 59.8 & 17.54 & 7.08 & $4.23 e+9$ & $\ldots$ & $\ldots$ \\
\hline F04406+2748 & 1FGL J0440.6+2748 & B2 $0437+27 B$ & 04:40:50.369 & $27: 50: 46.79$ & bzb & SJET & $\ldots$ & 31 & 25.29 & 0.00 & $3.19 \mathrm{e}+9$ & $\ldots$ & $\ldots$ \\
\hline $\mathrm{F} 04486+112 \mathrm{~A}$ & 1FGL J0448.6+1118 & CRATES J0448+1127 & 04:48:50.410 & $11: 27: 28.58$ & bzq & LJET & 1.369 & 383 & 60.13 & 23.11 & $1.23 \mathrm{e}+10$ & 28 & -36 \\
\hline F04486+112B & 1FGL J0448.6+1118 & PKS $0446+11$ & 04:49:07.670 & $11: 21: 28.58$ & bzb & SJET & $1.207^{\mathrm{b}}$ & 1260 & 60.13 & 23.11 & $1.24 \mathrm{e}+11$ & $\ldots$ & $\ldots$ \\
\hline F05092+1015 & 1FGL J0509.2+1015 & PKS 0506+101 & 05:09:27.461 & 10:11:44.59 & bzq & LJET & 0.621 & 310 & 53.24 & 22.08 & $4.00 \mathrm{e}+10$ & $\ldots$ & $\ldots$ \\
\hline F05100+180A & 1FGL J0510.0+1800 & CRATES J0509+1806 & 05:09:42.910 & $18: 06: 30.31$ & agu & LJET & $\ldots$ & 44.8 & 32.38 & 12.39 & $8.80 \mathrm{e}+9$ & 25 & 3 \\
\hline F05100+180B & 1FGL J0510.0+1800 & PKS 0507+17 & 05:10:02.369 & 18:00:41.58 & bzq & LJET & 0.416 & 441 & 32.38 & 12.39 & $3.63 e+10$ & 22 & -3 \\
\hline F05310+1331 & 1FGL J0531.0+1331 & PKS 0528+134 & 05:30:56.419 & 13:31:55.09 & bzq & LJET & 2.07 & 2220 & 146.45 & 13.71 & $1.34 \mathrm{e}+11$ & $\ldots$ & $\ldots$ \\
\hline F06072+4739 & 1FGL J0607.2+4739 & CGRaBS J0607+4739 & $06: 07: 23.249$ & $47: 39: 47.02$ & bzb & LJET & $\ldots$ & 226 & 32.89 & 13.16 & $3.51 \mathrm{e}+10$ & $\ldots$ & $\ldots$ \\
\hline
\end{tabular}




\begin{tabular}{|c|c|c|c|c|c|c|c|c|c|c|c|c|c|}
\hline Source Name & 1FGL Name & Alt. Name & R.A. & Decl. & $\begin{array}{l}\text { Opt. } \\
\text { Type }\end{array}$ & Class & $z$ & $S_{5}$ & $\begin{array}{l}\text { LAT } \\
\text { Flux }\end{array}$ & $\begin{array}{l}\Delta \text { LAT } \\
\text { Flux }\end{array}$ & Core $T_{B}$ & $\begin{array}{l}\text { Open. } \\
\text { Ang. }\end{array}$ & $\Delta$ P.A. \\
\hline F06127+4120 & 1FGL J0612.7+4120 & B3 0609+413 & 06:12:51.190 & $41: 22: 37.42$ & bzb & LJET & $\ldots$ & 296 & 39.74 & 13.60 & $5.21 \mathrm{e}+10$ & $\ldots$ & $\ldots$ \\
\hline F06169+5701 & 1FGL J0616.9+5701 & CRATES J0617+5701 & $06: 17: 16.920$ & 57:01:16.39 & bzb & LJET & $\ldots$ & 256 & 16.24 & 5.89 & $8.23 e+10$ & $\ldots$ & $\ldots$ \\
\hline F06254+4440 & 1FGL J0625.4+4440 & CGRaBS J0625+4440 & $06: 25: 18.259$ & 44:40:01.60 & bzb & PS & $\ldots$ & 162 & 21.27 & 8.33 & $2.25 e+11$ & $\ldots$ & $\ldots$ \\
\hline F06399+7325 & 1FGL J0639.9+7325 & CGRaBS J0639+7324 & 06:39:21.960 & $73: 24: 58.00$ & bzq & LJET & 1.854 & 612 & 39.52 & 17.33 & $4.73 e+10$ & 14 & 4 \\
\hline F06507+2503 & 1FGL J0650.7+2503 & 1ES $0647+250$ & 06:50:46.490 & 25:02:59.60 & bzb & PS & 0.203 & 21.5 & 16.73 & 5.54 & $3.49 \mathrm{e}+9$ & $\ldots$ & $\ldots$ \\
\hline F06544+5042 & 1FGL J0654.4+5042 & CGRaBS J0654+5042 & 06:54:22.090 & $50: 42: 23.90$ & agu & SJET & & 240 & 42.02 & 7.63 & $2.31 \mathrm{e}+10$ & $\ldots$ & $\ldots$ \\
\hline F06543+4514 & 1FGL J0654.3+4514 & B3 $0650+453$ & $06: 54: 23.710$ & $45: 14: 23.50$ & bzq & PS & 0.933 & 218 & 115.24 & 8.96 & $1.67 \mathrm{e}+10$ & $\ldots$ & $\ldots$ \\
\hline F07114+4731 & 1FGL J0711.4+4731 & B3 $0707+476$ & 07:10:46.111 & $47: 32: 11.11$ & bzb & LJET & $1.292^{\mathrm{b}}$ & 620 & 37.32 & 15.00 & $6.57 \mathrm{e}+10$ & $\ldots$ & $\ldots$ \\
\hline F07127+5033 & 1FGL J0712.7+5033 & CGRaBS J0712+5033 & 07:12:43.639 & $50: 33: 22.68$ & bzb & PS & & 252 & 29.25 & 10.21 & $6.14 \mathrm{e}+10$ & $\ldots$ & $\ldots$ \\
\hline F07193+3306 & 1FGL J0719.3+3306 & B2 $0716+33$ & 07:19:19.421 & 33:07:09.70 & bzq & PS & 0.779 & 383 & 70.34 & 7.31 & $9.91 e+10$ & $\ldots$ & $\ldots$ \\
\hline F07219+7120 & 1FGL J0721.9+7120 & CGRaBS J0721+7120 & 07:21:53.450 & $71: 20: 36.38$ & bzb & SJET & 0.31 & 1340 & 149.23 & 8.47 & $7.42 \mathrm{e}+11$ & $\ldots$ & $\ldots$ \\
\hline F07253+1431 & 1FGL J0725.3+1431 & $4 C+14.23$ & 07:25:16.810 & $14: 25: 13.69$ & bzq & LJET & 1.038 & 688 & 37.66 & 7.77 & $3.80 \mathrm{e}+10$ & 37 & -54 \\
\hline F07382+1741 & 1FGL J0738.2+1741 & PKS 0735+178 & 07:38:07.390 & $17: 42: 19.01$ & bzb & LJET & 0.424 & 644 & 43.78 & 13.96 & $1.40 \mathrm{e}+10$ & 31 & 10 \\
\hline J07426+5444 & 1FGL J0742.2+5443 & CRATES J0742+5444 & 07:42:39.7904 & $54: 44: 24.679$ & bzq & PS & 0.723 & 220 & 59.25 & 24.76 & $4.48 \mathrm{e}+11$ & $\ldots$ & $\ldots$ \\
\hline J07464+2549 & 1FGL J0746.6+2548 & B2 $0743+25$ & $07: 46: 25.8753$ & $25: 49: 02.146$ & bzq & SJET & 2.979 & 564 & 42.28 & 7.88 & $3.79 \mathrm{e}+11$ & $\ldots$ & $\ldots$ \\
\hline F07506+1235 & 1FGL J0750.6+1235 & PKS 0748+126 & 07:50:52.051 & $12: 31: 04.80$ & bzq & LJET & 0.889 & 3390 & 37.46 & 7.57 & $3.30 \mathrm{e}+11$ & $\ldots$ & $\ldots$ \\
\hline J07530+5352 & 1FGL J0752.8+5353 & $4 C+54.15$ & 07:53:01.3847 & 53:52:59.636 & bzb & PS & 0.2 & 485 & 27.01 & 11.32 & $3.76 e+10$ & $\ldots$ & $\ldots$ \\
\hline$J 08053+6144$ & 1FGL J0806.2+6148 & CGRaBS J0805+6144 & 08:05:18.1778 & 61:44:23.704 & bzq & SJET & 3.033 & 828 & 36.04 & 8.88 & $5.28 \mathrm{e}+10$ & $\ldots$ & $\ldots$ \\
\hline J08096+3455 & 1FGL J0809.4+3455 & B2 $0806+35$ & 08:09:38.8868 & $34: 55: 37.248$ & bzb & LJET & 0.082 & 58.6 & 9.00 & 3.34 & $1.90 \mathrm{e}+10$ & $\ldots$ & $\ldots$ \\
\hline J08098+5218 & 1FGL J0809.5+5219 & CRATES J0809+5218 & 08:09:49.1899 & $52: 18: 58.252$ & bzb & LJET & 0.138 & 108 & 43.03 & 7.77 & $2.78 \mathrm{e}+9$ & 50 & 20 \\
\hline J08146+6431 & 1FGL J0815.0+6434 & CGRaBS J0814+6431 & $08: 14: 39.1912$ & 64:31:22.04 & bzb & LJET & $\ldots$ & 102 & 32.76 & 12.91 & $7.31 \mathrm{e}+10$ & $\ldots$ & $\ldots$ \\
\hline J08163+5739 & 1FGL J0816.7+5739 & BZB J0816+5739 & $08: 16: 23.8223$ & 57:39:09.509 & bzb & CPLX & & 21.5 & 15.19 & 5.65 & $4.77 \mathrm{e}+8$ & $\ldots$ & $\ldots$ \\
\hline J08182+4222 & 1FGL J0818.2+4222 & B3 0814+425 & 08:18:15.9995 & 42:22:45.408 & bzb & SJET & $0.53^{\mathrm{b}}$ & 1660 & 108.47 & 8.38 & $3.17 \mathrm{e}+11$ & $\ldots$ & $\ldots$ \\
\hline J08247+5552 & 1FGL J0825.0+5555 & OJ 535 & $08: 24: 47.2364$ & $55: 52: 42.662$ & bzq & LJET & 1.417 & 971 & 62.43 & 8.92 & $3.80 \mathrm{e}+10$ & 17 & -11 \\
\hline $\mathrm{J} 08308+2410^{\mathrm{c}}$ & 1FGL J0830.5+2407 & OJ 248 & $08: 30: 52.0855$ & $24: 10: 59.818$ & bzq & SJET & 0.94 & 1110 & 65.29 & 8.21 & $4.14 \mathrm{e}+11$ & $\ldots$ & $\ldots$ \\
\hline J08338+4224 & 1FGL J0834.4+4221 & B3 $0830+425$ & $08: 33: 53.8852$ & 42:24:01.859 & bzq & SJET & 0.249 & 249 & 31.44 & 7.80 & $3.90 \mathrm{e}+10$ & $\ldots$ & $\ldots$ \\
\hline F08422+7054 & 1FGL J0842.2+7054 & $4 \mathrm{C}+71.07$ & $08: 41: 24.360$ & $70: 53: 42.22$ & bzq & LJET & 2.218 & 1680 & 61.94 & 8.92 & $9.87 \mathrm{e}+9$ & 9 & -19 \\
\hline F08499+4852 & 1FGL J0849.9+4852 & CRATES J0850+4854 & 08:50:00.530 & 48:54:52.60 & agu & LJET & & 60.1 & 29.87 & 6.99 & $1.20 \mathrm{e}+9$ & 54 & 121 \\
\hline $\mathrm{J} 08548+2006^{\mathrm{c}}$ & 1FGL J0854.8+2006 & OJ 287 & 08:54:48.8741 & 20:06:30.639 & bzb & SJET & 0.306 & 2380 & 63.31 & 13.83 & $3.17 \mathrm{e}+11$ & $\ldots$ & $\ldots$ \\
\hline J08566+2057 & 1FGL J0856.6+2103 & CRATES J0850+2057 & 08:56:39.7398 & 20:57:43.426 & bzq & LJET & $0.539^{\mathrm{b}}$ & 45.1 & 39.89 & 17.57 & $1.28 \mathrm{e}+10$ & 16 & 10 \\
\hline J08569+2111 & 1FGL J0856.6+2103 & OJ 290 & $08: 56: 57.2424$ & 21:11:43.64 & bzq & LJET & 2.098 & 365 & 39.89 & 17.57 & $8.24 \mathrm{e}+9$ & $\ldots$ & $\ldots$ \\
\hline F09055+1356 & 1FGL J0905.5+1356 & CRATES J0905+1358 & 09:05:34.990 & 13:58:06.31 & agu & SJET & $\ldots$ & 48.5 & 18.39 & 7.21 & $7.33 \mathrm{e}+9$ & $\ldots$ & $\ldots$ \\
\hline J09106+3329 & 1FGL J0910.7+3332 & Ton 1015 & 09:10:37.0354 & $33: 29: 24.418$ & bzb & LJET & 0.354 & 130 & 22.76 & 9.04 & $4.04 \mathrm{e}+10$ & $\ldots$ & $\ldots$ \\
\hline J09121+4126 & 1FGL J0912.3+4127 & B3 0908+416B & $09: 12: 11.6174$ & 41:26:09.356 & bzq & LJET & 2.563 & 132 & 18.51 & 7.61 & $3.95 e+9$ & $\ldots$ & $\ldots$ \\
\hline J09158+2933 & 1FGL J0915.7+2931 & B2 0912+29 & 09:15:52.4014 & 29:33:23.982 & bzb & LJET & & 80 & 22.64 & 8.34 & $1.30 \mathrm{e}+10$ & $\ldots$ & $\ldots$ \\
\hline J09209+4441 & 1FGL J0920.9+4441 & В3 $0917+449$ & 09:20:58.4599 & 44:41:53.988 & bzq & LJET & 2.19 & 1020 & 242.01 & 9.44 & $3.59 \mathrm{e}+10$ & 44 & -159 \\
\hline J09216+6215 & 1FGL J0919.6+6216 & OK 630 & $09: 21: 36.2322$ & $62: 15: 52.185$ & bzq & LJET & 1.446 & 1410 & 35.59 & 7.95 & $1.59 \mathrm{e}+11$ & $\ldots$ & $\ldots$ \\
\hline J09235+4125 & 1FGL J0923.2+4121 & B3 $0920+416$ & 09:23:31.3037 & $41: 25: 27.429$ & agn & LJET & 0.028 & 220 & 40.61 & 10.01 & $2.97 \mathrm{e}+10$ & 10 & 1 \\
\hline J09238+2815 & 1FGL J0924.2+2812 & B2 $0920+28$ & $09: 23: 51.522$ & $28: 15: 24.966$ & bzq & PS & 0.744 & 899 & 44.39 & 19.61 & $1.95 \mathrm{e}+11$ & $\ldots$ & $\ldots$ \\
\hline J09292+5013 & 1FGL J0929.4+5000 & CRATES J0929+5013 & 09:29:15.4401 & 50:13:35.982 & bzb & LJET & $0.37039^{b}$ & 430 & 21.40 & 9.29 & $4.81 \mathrm{e}+10$ & & $\ldots$ \\
\hline
\end{tabular}




\begin{tabular}{|c|c|c|c|c|c|c|c|c|c|c|c|c|c|}
\hline Source Name & 1FGL Name & Alt. Name & R.A. & Decl. & $\begin{array}{l}\text { Opt. } \\
\text { Type }\end{array}$ & Class & $z$ & $S_{5}$ & $\begin{array}{l}\text { LAT } \\
\text { Flux }\end{array}$ & $\begin{array}{l}\Delta \text { LAT } \\
\text { Flux }\end{array}$ & Core $T_{B}$ & $\begin{array}{l}\text { Open. } \\
\text { Ang. }\end{array}$ & $\Delta$ P.A. \\
\hline J09341+3926 & 1FGL J0934.5+3929 & CGRaBS J0934+3926 & 09:34:06.674 & 39:26:32.125 & bzb & PS & $0.044^{b}$ & 108 & 19.72 & 7.73 & $2.89 \mathrm{e}+10$ & $\ldots$ & $\ldots$ \\
\hline J09372+5008 & 1FGL J0937.7+5005 & CGRaBS J0937+5008 & $09: 37: 12.3257$ & 50:08:52.082 & bzq & PS & 0.276 & 324 & 52.33 & 11.77 & $1.05 \mathrm{e}+11$ & $\ldots$ & $\ldots$ \\
\hline J09418+2728 & 1FGL J0941.2+2722 & CGRaBS J0941+2728 & 09:41:48.1135 & $27: 28: 38.818$ & bzq & PS & 1.306 & 235 & 18.15 & 7.24 & $1.46 \mathrm{e}+10$ & $\ldots$ & $\ldots$ \\
\hline F09456+5754 & 1FGL J0945.6+5754 & CRATES J0945+5757 & $09: 45: 42.240$ & $57: 57: 47.70$ & bzb & LJET & 0.229 & 42.9 & 17.96 & 7.22 & $1.64 \mathrm{e}+10$ & $\ldots$ & $\ldots$ \\
\hline F09466+1012 & 1FGL J0946.6+1012 & CRATES J0946+1017 & 09:46:35.071 & 10:17:06.11 & bzq & SJET & 1.007 & 245 & 28.22 & 11.71 & $2.42 \mathrm{e}+10$ & $\ldots$ & $\ldots$ \\
\hline J09496+1752 & 1FGL J0949.8+1757 & CRATES J0949+1752 & $09: 49: 39.7634$ & $17: 52: 49.432$ & bzq & LJET & 0.693 & 207 & 18.85 & 0.00 & $1.50 \mathrm{e}+11$ & $\ldots$ & $\ldots$ \\
\hline F09498+1757 & 1FGL J0949.8+1757 & CRATES J0950+1804 & 09:50:00.310 & 18:04:18.70 & agu & SJET & $0.69327^{\mathrm{b}}$ & 37.2 & 18.85 & 0.00 & $1.43 \mathrm{e}+9$ & $\ldots$ & $\ldots$ \\
\hline F09565+6938 & 1FGL J0956.5+6938 & M 82 & 09:55:52.726 & $69: 40: 45.77$ & $\operatorname{sbg}$ & PS & $0.000677^{\mathrm{b}}$ & 14 & 38.40 & 16.23 & $3.39 \mathrm{e}+9$ & $\ldots$ & $\ldots$ \\
\hline J09568+2515 & 1FGL J0956.9+2513 & B2 $0954+25 A$ & 09:56:49.8747 & $25: 15: 16.047$ & bzq & PS & 0.712 & 645 & 28.07 & 11.59 & $7.88 \mathrm{e}+9$ & $\ldots$ & $\ldots$ \\
\hline J09576+5522 & 1FGL J0957.7+5523 & $4 C+55.17$ & 09:57:38.1837 & $55: 22: 57.74$ & bzq & LJET & 0.896 & 580 & 106.45 & 7.43 & $9.88 \mathrm{e}+8$ & 68 & -1 \\
\hline F10001+6539 & 1FGL J1000.1+6539 & CGRaBS J0958+6533 & 09:58:47.251 & 65:33:54.79 & bzb & LJET & 0.367 & 1450 & 28.21 & 11.72 & $5.07 \mathrm{e}+11$ & $\ldots$ & $\ldots$ \\
\hline F10127+2440 & 1FGL J1012.7+2440 & CRATES J1012+2439 & $10: 12: 41.381$ & $24: 39: 23.40$ & bzq & SJET & 1.805 & 63.9 & 49.66 & 6.81 & $5.56 \mathrm{e}+9$ & $\ldots$ & $\ldots$ \\
\hline J10150+4926 & 1FGL J1015.1+4927 & 1ES $1011+496$ & 10:15:04.1336 & 49:26:00.704 & bzb & LJET & 0.2 & 201 & 63.31 & 6.16 & $7.98 \mathrm{e}+9$ & $\ldots$ & $\ldots$ \\
\hline J10330+4116 & 1FGL J1033.2+4116 & B3 $1030+415$ & 10:33:03.7086 & 41:16:06.234 & bzq & LJET & 1.117 & 1290 & 29.30 & 6.86 & $2.22 \mathrm{e}+12$ & $\ldots$ & $\ldots$ \\
\hline J10338+6051 & 1FGL J1033.8+6048 & CGRaBS J1033+6051 & $10: 33: 51.427$ & 60:51:07.342 & bzq & SJET & 1.401 & 188 & 50.15 & 7.02 & $1.55 \mathrm{e}+10$ & $\ldots$ & $\ldots$ \\
\hline F10377+5711 & 1FGL J1037.7+5711 & CRATES J1037+5711 & $10: 37: 44.311$ & $57: 11: 55.61$ & bzb & PS & & 61.8 & 30.14 & 10.02 & $1.73 e+10$ & $\ldots$ & $\ldots$ \\
\hline J10431+2408 & 1FGL J1043.1+2404 & B2 $1040+24 \mathrm{~A}$ & 10:43:09.0347 & $24: 08: 35.43$ & bzb & SJET & 0.56 & 738 & 18.02 & 6.92 & $1.26 \mathrm{e}+11$ & $\ldots$ & $\ldots$ \\
\hline F10487+8054 & 1FGL J1048.7+8054 & CGRaBS J1044+8054 & 10:44:23.071 & $80: 54: 39.38$ & bzq & LJET & 1.26 & 727 & 57.94 & 8.73 & $3.32 \mathrm{e}+10$ & 3 & -10 \\
\hline F10485+7239 & 1FGL J1048.5+7239 & CRATES J1047+7238 & 10:47:47.520 & 72:38:13.02 & agu & LJET & 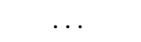 & 61 & 44.18 & 19.09 & $1.60 \mathrm{e}+10$ & $\ldots$ & $\ldots$ \\
\hline F10488+7145 & 1FGL J1048.8+7145 & CGRaBS J1048+7143 & $10: 48: 27.619$ & 71:43:35.90 & bzq & PS & 1.15 & 1170 & 59.88 & 26.71 & $1.09 \mathrm{e}+11$ & $\ldots$ & $\ldots$ \\
\hline J10586+5628 & 1FGL J1058.6+5628 & CGRaBS J1058+5628 & $10: 58: 37.7261$ & $56: 28: 11.18$ & bzb & LJET & 0.143 & 159 & 56.29 & 6.84 & $1.93 \mathrm{e}+10$ & $\ldots$ & $\ldots$ \\
\hline $\mathrm{J} 11044+3812$ & 1FGL J1104.4+3812 & Mkn 421 & 11:04:27.3145 & $38: 12: 31.794$ & bzb & LJET & 0.03 & 262 & 170.63 & 7.25 & $8.35 \mathrm{e}+10$ & $\ldots$ & $\ldots$ \\
\hline $\mathrm{J} 11061+2812$ & 1FGL J1106.5+2809 & CRATES J1106+2812 & 11:06:07.2592 & 28:12:47.045 & agu & PS & $0.847^{b}$ & 227 & 34.63 & 15.03 & $2.25 \mathrm{e}+11$ & $\ldots$ & $\ldots$ \\
\hline J11126+3446 & 1FGL J1112.8+3444 & CRATES J1112+3446 & $11: 12: 38.7673$ & 34:46:39.124 & bzq & LJET & 1.949 & 197 & 33.70 & 13.80 & $6.37 \mathrm{e}+10$ & $\ldots$ & $\ldots$ \\
\hline F11171+2013 & 1FGL J1117.1+2013 & CRATES J1117+2014 & 11:17:06.259 & 20:14:07.40 & bzb & LJET & 0.138 & 36.5 & 11.66 & 3.57 & $1.28 \mathrm{e}+10$ & $\ldots$ & $\ldots$ \\
\hline $\mathrm{J} 11240+2336$ & 1FGL J1123.9+2339 & OM 235 & 11:24:02.7109 & $23: 36: 45.876$ & bzb & SJET & & 362 & 23.95 & 9.65 & $6.96 e+10$ & $\ldots$ & $\ldots$ \\
\hline F11366+7009 & 1FGL J1136.6+7009 & Mkn 180 & $11: 36: 26.410$ & 70:09:27.32 & bzb & LJET & 0.045 & 136 & 13.12 & 4.46 & $2.51 \mathrm{e}+10$ & $\ldots$ & $\ldots$ \\
\hline J11421+1547 & 1FGL J1141.8+1549 & CRATES J1142+1547 & 11:42:07.7378 & $15: 47: 54.202$ & agu & LJET & & 139 & 12.87 & 5.19 & $9.15 e+10$ & 28 & 5 \\
\hline J11469+3958 & 1FGL J1146.8+4004 & B2 $1144+40$ & 11:46:58.2987 & 39:58:34.307 & bzq & PS & 1.089 & 603 & 46.91 & 20.17 & $2.85 \mathrm{e}+11$ & $\ldots$ & $\ldots$ \\
\hline J11503+2417 & 1FGL J1150.2+2419 & B2 $1147+24$ & 11:50:19.2146 & 24:17:53.852 & bzb & LJET & 0.2 & 621 & 29.11 & 11.81 & $4.47 \mathrm{e}+10$ & 20 & -8 \\
\hline J11514+5859 & 1FGL J1151.6+5857 & CRATES J1151+5859 & $11: 51: 24.6554$ & 58:59:17.552 & bzb & SJET & & 61.7 & 18.22 & 7.12 & $3.88 \mathrm{e}+9$ & $\ldots$ & $\ldots$ \\
\hline $\mathrm{J} 11540+6022$ & 1FGL J1152.1+6027 & CRATES J1154+6022 & 11:54:04.5339 & $60: 22: 20.785$ & $\ldots$ & PS & $\ldots$ & 232 & 46.21 & 20.76 & $3.93 e+11$ & $\ldots$ & $\ldots$ \\
\hline $\mathrm{J} 11595+2914^{\mathrm{c}}$ & 1FGL J1159.4+2914 & $4 C+29.45$ & $11: 59: 31.8338$ & $29: 14: 43.823$ & bzq & LJET & 0.729 & 1250 & 115.40 & 7.84 & $2.36 \mathrm{e}+12$ & $\ldots$ & $\ldots$ \\
\hline J12030+6031 & 1FGL J1202.9+6032 & CRATES J1203+6031 & 12:03:03.5094 & 60:31:19.129 & agn & LJET & 0.065 & 145 & 44.79 & 20.07 & $2.75 e+10$ & $\ldots$ & $\ldots$ \\
\hline J12089+5441 & 1FGL J1209.3+5444 & CRATES J1208+5441 & 12:08:54.2583 & $54: 41: 58.19$ & bzq & PS & 1.344 & 252 & 35.45 & 15.20 & $2.28 \mathrm{e}+11$ & $\ldots$ & $\ldots$ \\
\hline J12093+4119 & 1FGL J1209.4+4119 & B3 1206+416 & $12: 09: 22.7851$ & 41:19:41.36 & bzb & LJET & $0.377^{b}$ & 131 & 22.25 & 9.57 & $2.91 \mathrm{e}+10$ & $\ldots$ & $\ldots$ \\
\hline J12098+1810 & 1FGL J1209.7+1806 & CRATES J1209+1810 & 12:09:51.7649 & 18:10:06.796 & bzq & SJET & 0.845 & 140 & 19.72 & 7.97 & $3.19 \mathrm{e}+10$ & $\ldots$ & $\ldots$ \\
\hline J12178+3007 & 1FGL J1217.7+3007 & B2 $1215+30$ & $12: 17: 52.0838$ & 30:07:00.625 & bzb & LJET & 0.13 & 261 & 83.01 & 31.55 & $7.36 \mathrm{e}+10$ & $\ldots$ & $\ldots$ \\
\hline F12215+7106 & 1FGL J1221.5+7106 & CRATES J1220+7105 & 12:20:03.631 & 71:05:31.09 & bzq & SJET & 0.451 & 183 & 10.07 & 3.84 & $9.96 \mathrm{e}+10$ & $\ldots$ & $\ldots$ \\
\hline J12201+3431 & 1FGL J1220.2+3432 & CGRaBS J1220+3431 & 12:20:08.2902 & $34: 31: 21.711$ & bzb & LJET & $0.643^{\mathrm{b}}$ & 129 & 11.03 & 4.65 & $1.90 \mathrm{e}+10$ & $\ldots$ & $\ldots$ \\
\hline
\end{tabular}




\begin{tabular}{|c|c|c|c|c|c|c|c|c|c|c|c|c|c|}
\hline Source Name & 1FGL Name & Alt. Name & R.A. & Decl. & $\begin{array}{l}\text { Opt. } \\
\text { Type }\end{array}$ & Class & $z$ & $S_{5}$ & $\begin{array}{l}\text { LAT } \\
\text { Flux }\end{array}$ & $\begin{array}{c}\Delta \text { LAT } \\
\text { Flux }\end{array}$ & Core $T_{B}$ & $\begin{array}{l}\text { Open. } \\
\text { Ang. }\end{array}$ & $\Delta$ P.A. \\
\hline $\mathrm{J} 12215+2813$ & 1FGL J1221.5+2814 & W Com & $12: 21: 31.6936$ & 28:13:58.497 & bzb & LJET & 0.102 & 320 & 76.04 & 11.97 & $3.64 \mathrm{e}+10$ & & $\ldots$ \\
\hline F12248+8044 & 1FGL J1224.8+8044 & CRATES J1223+8040 & $12: 23: 40.500$ & 80:40:04.30 & bzb & LJET & & 450 & 25.48 & 10.12 & $4.01 \mathrm{e}+10$ & 27 & -1 \\
\hline $\mathrm{J} 12248+4335$ & 1FGL J1225.8+4336 & B3 $1222+438$ & $12: 24: 51.5074$ & $43: 35: 19.276$ & agu & LJET & $1.07491^{\mathrm{b}}$ & 207 & 36.15 & 15.51 & $3.15 \mathrm{e}+10$ & $\ldots$ & $\ldots$ \\
\hline $\mathrm{J} 12249+2122^{\mathrm{c}}$ & 1FGL J1224.7+2121 & $4 C+21.35$ & $12: 24: 54.4600$ & $21: 22: 46.438$ & bzq & LJET & 0.435 & 746 & 69.70 & 7.78 & $3.97 \mathrm{e}+11$ & 81 & 5 \\
\hline $\mathrm{J} 12269+4340$ & 1FGL J1225.8+4336 & B3 $1224+439$ & $12: 26: 57.9051$ & $43: 40: 58.438$ & bzq & SJET & 2.002 & 85 & 36.15 & 15.51 & $8.58 \mathrm{e}+9$ & & $\ldots$ \\
\hline $\mathrm{J} 12302+2518$ & 1FGL J1230.4+2520 & ON 246 & $12: 30: 14.0935$ & $25: 18: 07.145$ & bzb & LJET & 0.135 & 206 & 27.43 & 11.46 & $1.97 \mathrm{e}+10$ & 33 & -3 \\
\hline $\mathrm{F} 12316+2850$ & 1FGL J1231.6+2850 & B2 $1229+29$ & $12: 31: 43.579$ & $28: 47: 49.81$ & bzb & LJET & 0.236 & 86.3 & 25.44 & 8.13 & $1.33 \mathrm{e}+10$ & 17 & 7 \\
\hline $\mathrm{F} 12431+3627$ & 1FGL J1243.1+3627 & B2 $1240+36$ & $12: 43: 12.739$ & $36: 27: 43.99$ & bzb & SJET & $1.0654^{\mathrm{b}}$ & 59.6 & 24.30 & 9.36 & $8.77 e+9$ & $\ldots$ & $\ldots$ \\
\hline $\mathrm{J} 12483+5820$ & 1FGL J1248.2+5820 & CGRaBS J1248+5820 & $12: 48: 18.784$ & $58: 20: 28.725$ & bzb & LJET & $0.847^{\mathrm{b}}$ & 124 & 61.70 & 6.81 & $3.85 \mathrm{e}+10$ & 36 & 2 \\
\hline $\mathrm{J} 12531+5301$ & 1FGL J1253.0+5301 & CRATES J1253+5301 & $12: 53: 11.9232$ & $53: 01: 11.741$ & bzb & LJET & & 240 & 35.44 & 6.32 & $2.91 \mathrm{e}+10$ & 10 & 3 \\
\hline $\mathrm{J} 12579+3229$ & 1FGL J1258.3+3227 & B2 $1255+32$ & $12: 57: 57.2313$ & $32: 29: 29.321$ & bzq & LJET & 0.806 & 467 & 21.96 & 0.00 & $3.49 \mathrm{e}+10$ & $\ldots$ & $\ldots$ \\
\hline $\mathrm{J} 13030+2433$ & 1FGL J1303.0+2433 & CRATES J1303+2433 & 13:03:03.2143 & $24: 33: 55.684$ & bzb & PS & $0.993^{b}$ & 134 & 36.84 & 6.88 & $1.52 \mathrm{e}+11$ & $\ldots$ & $\ldots$ \\
\hline F13060+7852 & 1FGL J1306.0+7852 & CRATES J1305+7854 & 13:05:00.019 & $78: 54: 35.71$ & $\mathrm{agu}$ & SJET & & 213 & 17.53 & 6.82 & $1.82 \mathrm{e}+10$ & $\ldots$ & $\ldots$ \\
\hline $\mathrm{J} 13083+3546$ & 1FGL J1308.5+3550 & CGRaBS J1308+3546 & 13:08:23.7095 & $35: 46: 37.16$ & bzq & SJET & 1.055 & 309 & 41.59 & 16.49 & $3.25 \mathrm{e}+11$ & $\ldots$ & $\ldots$ \\
\hline F13092+1156 & 1FGL J1309.2+1156 & $4 C+12.46$ & 13:09:33.931 & $11: 54: 24.59$ & bzb & LJET & & 629 & 29.66 & 12.32 & $9.56 e+9$ & 33 & 8 \\
\hline $\mathrm{J} 13104+3220^{\mathrm{c}}$ & 1FGL J1310.6+3222 & B2 $1308+32$ & $13: 10: 28.6618$ & $32: 20: 43.790$ & bzq & LJET & 0.997 & 1240 & 135.55 & 8.98 & $6.57 e+11$ & 25 & -32 \\
\hline $\mathrm{J} 13127+4828$ & 1FGL J1312.4+4827 & CGRaBS J1312+4828 & 13:12:43.3508 & $48: 28: 30.928$ & bzq & PS & 0.501 & 66.7 & 31.29 & 12.84 & $1.62 \mathrm{e}+9$ & $\ldots$ & $\ldots$ \\
\hline $\mathrm{J} 13147+2348$ & 1FGL J1314.7+2346 & CRATES J1314+2348 & $13: 14: 43.8021$ & $23: 48: 26.701$ & bzb & LJET & & 123 & 35.58 & 15.30 & $2.22 \mathrm{e}+10$ & $\ldots$ & $\ldots$ \\
\hline $\mathrm{J} 13176+3425$ & 1FGL J1317.8+3425 & B2 $1315+34 \mathrm{~A}$ & $13: 17: 36.4935$ & $34: 25: 15.921$ & bzq & LJET & 1.05 & 260 & 18.93 & 7.43 & $4.47 \mathrm{e}+10$ & $\ldots$ & $\ldots$ \\
\hline $\mathrm{J} 13211+2216$ & 1FGL J1321.1+2214 & CGRaBS J1321+2216 & $13: 21: 11.2041$ & $22: 16: 12.098$ & bzq & SJET & 0.943 & 244 & 34.18 & 14.92 & $2.14 \mathrm{e}+10$ & $\ldots$ & $\ldots$ \\
\hline $\mathrm{F} 13213+8310$ & 1FGL J1321.3+8310 & CRATES J1321+8316 & $13: 21: 45.590$ & 83:16:13.40 & agu & LJET & $1.024^{\mathrm{b}}$ & 232 & 27.88 & 11.62 & $1.32 \mathrm{e}+10$ & 9 & -9 \\
\hline $\mathrm{J} 13270+2210^{\mathrm{c}}$ & 1FGL J1326.6+2213 & B2 $1324+22$ & $13: 27: 00.8577$ & $22: 10: 50.150$ & bzq & SJET & 1.4 & 1120 & 54.09 & 11.78 & $1.22 \mathrm{e}+12$ & $\ldots$ & $\ldots$ \\
\hline $\mathrm{J} 13307+5202$ & 1FGL J1331.0+5202 & CGRaBS J1330+5202 & $13: 30: 42.5962$ & $52: 02: 15.448$ & agn & LJET & 0.688 & 148 & 41.52 & 0.00 & $4.50 \mathrm{e}+10$ & $\ldots$ & $\ldots$ \\
\hline $\mathrm{J} 13327+4722$ & 1FGL J1332.9+4728 & B3 $1330+476$ & $13: 32: 45.2413$ & $47: 22: 22.653$ & bzq & PS & 0.669 & 298 & 32.08 & 14.24 & $1.30 \mathrm{e}+11$ & $\ldots$ & $\ldots$ \\
\hline $\mathrm{J} 13338+5057$ & 1FGL J1333.2+5056 & CLASS J1333+5057 & $13: 33: 53.7823$ & $50: 57: 35.914$ & agu & PS & $1.362^{\mathrm{b}}$ & 48.4 & 64.33 & 27.63 & $1.46 \mathrm{e}+10$ & $\ldots$ & $\ldots$ \\
\hline $\mathrm{J} 13455+4452$ & 1FGL J1345.4+4453 & B3 $1343+451$ & $13: 45: 33.1685$ & $44: 52: 59.581$ & bzq & SJET & 2.534 & 333 & 52.18 & 21.04 & $2.11 \mathrm{e}+11$ & $\ldots$ & $\ldots$ \\
\hline $\mathrm{J} 13508+3034$ & 1FGL J1351.0+3035 & B2 1348+30B & $13: 50: 52.7333$ & $30: 34: 53.582$ & bzq & SJET & 0.714 & 251 & 14.81 & 5.25 & $3.29 e+10$ & $\ldots$ & $\ldots$ \\
\hline F13533+1434 & 1FGL J1353.3+1434 & PKS 1350+148 & $13: 53: 22.841$ & $14: 35: 39.30$ & bzb & LJET & & 177 & 24.77 & 10.48 & $9.44 \mathrm{e}+9$ & 32 & 29 \\
\hline $\mathrm{F} 13581+7646$ & 1FGL J1358.1+7646 & CGRaBS J1357+7643 & $13: 57: 55.370$ & $76: 43: 21.00$ & bzq & SJET & 1.585 & 425 & 40.70 & 17.83 & $8.58 \mathrm{e}+10$ & $\ldots$ & $\ldots$ \\
\hline $\mathrm{J} 13590+5544$ & 1FGL J1359.1+5539 & CRATES J1359+5544 & 13:59:05.7379 & $55: 44: 29.362$ & bzq & PS & 1.014 & 108 & 37.91 & 16.61 & $6.36 \mathrm{e}+10$ & $\ldots$ & $\ldots$ \\
\hline $\mathrm{J} 14270+2348$ & 1FGL J1426.9+2347 & PKS $1424+240$ & $14: 27: 00.3942$ & 23:48:00.045 & bzb & CPLX & & 199 & 69.51 & 7.92 & $1.21 \mathrm{e}+10$ & $\ldots$ & $\ldots$ \\
\hline $\mathrm{J} 14340+4203$ & 1FGL J1433.9+4204 & B3 $1432+422$ & $14: 34: 05.6956$ & 42:03:16.01 & bzq & SJET & 1.24 & 131 & 25.17 & 10.63 & $1.50 \mathrm{e}+10$ & $\ldots$ & $\ldots$ \\
\hline $\mathrm{J} 14366+2321$ & 1FGL J1436.9+2314 & PKS 1434+235 & $14: 36: 40.9873$ & $23: 21: 03.297$ & bzq & LJET & 1.545 & 600 & 24.69 & 10.78 & $2.79 e+11$ & $\ldots$ & $\ldots$ \\
\hline $\mathrm{J} 14388+3710$ & 1FGL J1438.7+3711 & B2 $1436+37 B$ & 14:38:53.6095 & $37: 10: 35.408$ & bzq & LJET & 2.401 & 263 & 33.44 & 15.00 & $2.31 \mathrm{e}+11$ & $\ldots$ & $\ldots$ \\
\hline F14387+3711 & 1FGL J1438.7+3711 & CRATES J1439+3712 & 14:39:20.580 & $37: 12: 02.81$ & bzq & LJET & 1.021 & 33.3 & 33.44 & 15.00 & $9.52 \mathrm{e}+9$ & $\ldots$ & $\ldots$ \\
\hline $\mathrm{F} 14438+2457$ & 1FGL J1443.8+2457 & PKS $1441+25$ & $14: 43: 56.890$ & $25: 01: 44.51$ & bzq & LJET & 0.939 & 286 & 20.05 & 7.96 & $1.15 \mathrm{e}+11$ & $\ldots$ & $\ldots$ \\
\hline $\mathrm{J} 14509+5201$ & 1FGL J1451.0+5204 & CLASS J1450+5201 & 14:50:59.9877 & 52:01:11.7 & bzb & PS & & 37.5 & 33.61 & 14.31 & $2.25 \mathrm{e}+10$ & $\ldots$ & $\ldots$ \\
\hline $\mathrm{J} 14544+5124$ & 1FGL J1454.6+5125 & CRATES J1454+5124 & 14:54:27.1247 & $51: 24: 33.734$ & bzb & LJET & $1.08^{\mathrm{b}}$ & 80.2 & 50.54 & 22.17 & $5.83 e+9$ & $\ldots$ & $\ldots$ \\
\hline F15044+1029 & 1FGL J1504.4+1029 & PKS 1502+106 & $15: 04: 24.979$ & 10:29:39.19 & bzq & LJET & 1.839 & 1030 & 1061.22 & 14.67 & $3.54 \mathrm{e}+10$ & 56 & -18 \\
\hline $\mathrm{J} 15061+3730$ & 1FGL J1505.8+3725 & B2 1504+37 & 15:06:09.5287 & $37: 30: 51.128$ & bzq & LJET & 0.674 & 630 & 31.13 & 12.90 & $8.54 \mathrm{e}+10$ & 11 & 3 \\
\hline
\end{tabular}


Table 1

\begin{tabular}{|c|c|c|c|c|c|c|c|c|c|c|c|c|c|}
\hline Source Name & 1FGL Name & Alt. Name & R.A. & Decl. & $\begin{array}{l}\text { Opt. } \\
\text { Type }\end{array}$ & Class & $z$ & $S_{5}$ & $\begin{array}{l}\text { LAT } \\
\text { Flux }\end{array}$ & $\begin{array}{c}\Delta \text { LAT } \\
\text { Flux }\end{array}$ & Core $T_{B}$ & $\begin{array}{c}\text { Open. } \\
\text { Ang. }\end{array}$ & $\Delta$ P.A. \\
\hline $\mathrm{J} 15169+1932$ & 1FGL J1516.9+1928 & PKS 1514+197 & $15: 16: 56.7985$ & 19:32:13.01 & bzb & LJET & $1.07^{\mathrm{b}}$ & 662 & 35.06 & 15.62 & $2.27 \mathrm{e}+11$ & $\ldots$ & $\ldots$ \\
\hline F15197+4216 & 1FGL J1519.7+4216 & B3 1518+423 & $15: 20: 39.720$ & $42: 11: 11.51$ & bzq & PS & 0.484 & 40.8 & 20.75 & 8.72 & $1.09 \mathrm{e}+10$ & $\ldots$ & $\ldots$ \\
\hline J15221+3144 & 1FGL J1522.1+3143 & B2 1520+31 & 15:22:09.9947 & 31:44:14.427 & bzq & CPLX & 1.487 & 462 & 411.49 & 10.60 & $1.12 \mathrm{e}+11$ & $\ldots$ & $\ldots$ \\
\hline J15396+2744 & 1FGL J1539.7+2747 & CGRaBS J1539+2744 & $15: 39: 39.141$ & 27:44:38.288 & bzq & SJET & 2.19 & 145 & 13.50 & 4.90 & $9.70 \mathrm{e}+10$ & $\ldots$ & $\ldots$ \\
\hline J15429+6129 & 1FGL J1542.9+6129 & CRATES J1542+6129 & $15: 42: 56.9464$ & 61:29:55.358 & bzb & LJET & & 97.8 & 65.53 & 6.88 & $2.38 \mathrm{e}+10$ & $\ldots$ & $\ldots$ \\
\hline F15534+1255 & 1FGL J1553.4+1255 & PKS 1551+130 & $15: 53: 32.700$ & $12: 56: 51.68$ & bzq & LJET & 1.308 & 658 & 119.49 & 13.66 & $1.21 \mathrm{e}+10$ & 29 & -157 \\
\hline F15557+1111 & 1FGL J1555.7+1111 & PG $1553+113$ & $15: 55: 43.039$ & $11: 11: 24.40$ & bzb & SJET & 0.36 & 160 & 77.62 & 22.73 & $6.35 e+10$ & $\ldots$ & $\ldots$ \\
\hline $\mathrm{J} 16046+5714$ & 1FGL J1604.3+5710 & CGRaBS J1604+5714 & $16: 04: 37.3568$ & 57:14:36.668 & bzq & LJET & 0.72 & 374 & 54.28 & 23.42 & $1.01 \mathrm{e}+10$ & 3 & 14 \\
\hline $\mathrm{J} 16071+1551$ & 1FGL J1607.1+1552 & $4 C+15.54$ & 16:07:06.4276 & $15: 51: 34.495$ & agn & LJET & 0.496 & 322 & 33.90 & 7.75 & $3.19 \mathrm{e}+10$ & 27 & 4 \\
\hline F16090+1031 & 1FGL J1609.0+1031 & $4 C+10.45$ & 16:08:46.200 & 10:29:07.80 & bzq & LJET & 1.226 & 1120 & 61.34 & 9.82 & $4.12 \mathrm{e}+10$ & $\ldots$ & $\ldots$ \\
\hline $\mathrm{J} 16136+3412^{\mathrm{c}}$ & 1FGL J1613.5+3411 & B2 $1611+34$ & $16: 13: 41.0633$ & 34:12:47.903 & bzq & LJET & 1.397 & 2960 & 22.67 & 9.88 & $3.58 \mathrm{e}+10$ & $\ldots$ & $\ldots$ \\
\hline J16160+4632 & 1FGL J1616.1+4637 & CRATES J1616+4632 & 16:16:03.7689 & $46: 32: 25.239$ & bzq & PS & 0.95 & 74.2 & 37.69 & 16.18 & $4.16 \mathrm{e}+10$ & $\ldots$ & $\ldots$ \\
\hline $\mathrm{F} 16302+5220$ & 1FGL J1630.2+5220 & CRATES J1630+5221 & $16: 30: 43.150$ & $52: 21: 38.59$ & bzb & PS & & 27.3 & 19.32 & 7.35 & $1.59 \mathrm{e}+9$ & $\ldots$ & $\ldots$ \\
\hline F16354+8228 & 1FGL J1635.4+8228 & NGC 6251 & $16: 32: 31.980$ & $82: 32: 16.40$ & agn & LJET & 0.025 & 637 & 45.51 & 18.77 & $1.41 \mathrm{e}+10$ & 1 & 2 \\
\hline J16377+4717 & 1FGL J1637.9+4707 & $4 C+47.44$ & $16: 37: 45.1338$ & 47:17:33.822 & bzq & LJET & 0.74 & 673 & 35.84 & 8.79 & $7.56 e+10$ & $\ldots$ & $\ldots$ \\
\hline F16410+1143 & 1FGL J1641.0+1143 & CRATES J1640+1144 & $16: 40: 58.889$ & $11: 44: 04.20$ & agn & LJET & 0.078 & 115 & 47.36 & 20.81 & $7.65 e+9$ & $\ldots$ & $\ldots$ \\
\hline J16475+4950 & 1FGL J1647.4+4948 & CGRaBS J1647+4950 & $16: 47: 34.9142$ & 49:50:00.586 & agn & LJET & 0.047 & 154 & 33.29 & 8.54 & $2.02 \mathrm{e}+10$ & $\ldots$ & $\ldots$ \\
\hline J16568+6012 & 1FGL J1656.9+6017 & CRATES J1656+6012 & $16: 56: 48.2475$ & $60: 12: 16.455$ & bzq & PS & 0.623 & 323 & 18.12 & 7.82 & $7.16 \mathrm{e}+10$ & $\ldots$ & $\ldots$ \\
\hline $\mathrm{F} 17001+6830$ & 1FGL J1700.1+6830 & CGRaBS J1700+6830 & 17:00:09.300 & 68:30:07.02 & bzq & PS & 0.301 & 372 & 55.99 & 7.32 & $7.28 \mathrm{e}+10$ & $\ldots$ & $\ldots$ \\
\hline J17096+4318 & 1FGL J1709.6+4320 & B3 $1708+433$ & 17:09:41.0876 & 43:18:44.547 & bzq & LJET & 1.027 & 140 & 42.84 & 17.10 & $2.19 \mathrm{e}+10$ & $\ldots$ & $\ldots$ \\
\hline F17192+1745 & 1FGL J1719.2+1745 & PKS 1717+177 & 17:19:13.049 & 17:45:06.41 & bzb & SJET & 0.137 & 642 & 44.76 & 15.61 & $1.66 \mathrm{e}+11$ & $\ldots$ & $\ldots$ \\
\hline F17225+1012 & 1FGL J1722.5+1012 & CRATES J1722+1013 & $17: 22: 44.580$ & $10: 13: 35.80$ & bzq & SJET & 0.732 & 386 & 59.14 & 26.13 & $1.93 \mathrm{e}+10$ & $\ldots$ & $\ldots$ \\
\hline J17240+4004 & 1FGL J1724.0+4002 & B2 $1722+40$ & $17: 24: 05.4301$ & 40:04:36.457 & agn & LJET & 1.049 & 435 & 47.16 & 8.97 & $3.32 \mathrm{e}+11$ & $\ldots$ & $\ldots$ \\
\hline F17250+1151 & 1FGL J1725.0+1151 & CGRaBS J1725+1152 & 17:25:04.339 & 11:52:15.49 & bzb & SJET & $0.018^{\mathrm{b}}$ & 63.3 & 54.96 & 23.25 & $4.47 \mathrm{e}+10$ & $\ldots$ & $\ldots$ \\
\hline J17274+4530 & 1FGL J1727.3+4525 & B3 $1726+455$ & $17: 27: 27.6472$ & $45: 30: 39.743$ & bzq & SJET & 0.714 & 1050 & 39.28 & 7.93 & $1.68 \mathrm{e}+12$ & $\ldots$ & $\ldots$ \\
\hline $\mathrm{J} 17283+5013$ & 1FGL J1727.9+5010 & I Zw187 & $17: 28: 18.6238$ & $50: 13: 10.48$ & bzb & LJET & 0.055 & 133 & 28.67 & 12.02 & $6.88 \mathrm{e}+9$ & 11 & 31 \\
\hline F17308+3716 & 1FGL J1730.8+3716 & CRATES J1730+3714 & $17: 30: 47.050$ & $37: 14: 55.10$ & bzb & SJET & & 54.9 & 24.08 & 9.56 & $1.91 \mathrm{e}+9$ & $\ldots$ & $\ldots$ \\
\hline $\mathrm{J} 17343+3857$ & 1FGL J1734.4+3859 & B2 $1732+38 \mathrm{~A}$ & $17: 34: 20.5821$ & 38:57:51.446 & bzq & PS & 0.976 & 872 & 99.48 & 10.85 & $1.22 \mathrm{e}+11$ & $\ldots$ & $\ldots$ \\
\hline J17425+5945 & 1FGL J1742.1+5947 & CRATES J1742+5945 & $17: 42: 32.0074$ & 59:45:06.729 & bzb & LJET & $\ldots$ & 118 & 27.81 & 7.02 & $1.58 \mathrm{e}+10$ & 1 & -1 \\
\hline F17442+1934 & 1FGL J1744.2+1934 & 1ES $1741+196$ & $17: 43: 57.830$ & 19:35:08.99 & bzb & SJET & 0.083 & 154 & 15.54 & 6.15 & $1.12 \mathrm{e}+10$ & $\ldots$ & $\ldots$ \\
\hline F17485+7004 & 1FGL J1748.5+7004 & CGRaBS J1748+7005 & $17: 48: 32.839$ & 70:05:50.78 & bzb & CPLX & 0.77 & 615 & 31.49 & 12.02 & $2.88 \mathrm{e}+10$ & $\ldots$ & $\ldots$ \\
\hline J17490+4321 & 1FGL J1749.0+4323 & B3 $1747+433$ & 17:49:00.3604 & $43: 21: 51.287$ & bzb & LJET & $\ldots$ & 373 & 35.44 & 14.39 & $6.46 e+10$ & $\ldots$ & $\ldots$ \\
\hline F17566+5524 & 1FGL J1756.6+5524a & CRATES J1757+5523 & $17: 57: 28.279$ & $55: 23: 11.90$ & bzb & LJET & 0.065 & 40.7 & 27.79 & 12.00 & $4.04 \mathrm{e}+9$ & $\ldots$ & $\ldots$ \\
\hline F18004+7827 & 1FGL J1800.4+7827 & CGRaBS J1800+7828 & 18:00:45.679 & 78:28:04.01 & bzb & LJET & 0.68 & 2370 & 64.41 & 7.67 & $1.28 \mathrm{e}+11$ & 12 & -8 \\
\hline F18070+6945 & 1FGL J1807.0+6945 & 3C 371 & 18:06:50.681 & 69:49:28.09 & bzb & LJET & 0.051 & 1200 & 75.93 & 12.26 & $4.46 \mathrm{e}+10$ & 6 & 14 \\
\hline F18096+2908 & 1FGL J1809.6+2908 & CRATES J1809+2910 & 18:09:45.389 & 29:10:19.88 & bzb & SJET & & 96.9 & 21.61 & 8.26 & $1.32 \mathrm{e}+11$ & $\ldots$ & $\ldots$ \\
\hline $\mathrm{F} 18134+3141$ & 1FGL J1813.4+3141 & B2 $1811+31$ & $18: 13: 35.210$ & $31: 44: 17.59$ & bzb & LJET & 0.117 & 81.3 & 30.48 & 11.57 & $1.31 \mathrm{e}+10$ & $\ldots$ & $\ldots$ \\
\hline F18240+5651 & 1FGL J1824.0+5651 & $4 \mathrm{C}+56.27$ & 18:24:07.070 & $56: 51: 01.51$ & bzb & LJET & $0.664^{\mathrm{b}}$ & 1060 & 64.59 & 10.22 & $1.82 \mathrm{e}+11$ & $\ldots$ & $\ldots$ \\
\hline F18485+3224 & 1FGL J1848.5+3224 & B2 $1846+32 \mathrm{~A}$ & 18:48:22.099 & $32: 19: 02.60$ & bzq & LJET & 0.798 & 322 & 74.19 & 15.38 & $3.55 \mathrm{e}+10$ & $\ldots$ & $\ldots$ \\
\hline F18493+6705 & 1FGL J1849.3+6705 & CGRaBS J1849+6705 & $18: 49: 16.080$ & $67: 05: 41.71$ & bzq & LJET & 0.657 & 1310 & 227.47 & 9.47 & $3.84 \mathrm{e}+11$ & $\ldots$ & $\ldots$ \\
\hline
\end{tabular}


Table 1
(Continued)

\begin{tabular}{|c|c|c|c|c|c|c|c|c|c|c|c|c|c|}
\hline Source Name & 1FGL Name & Alt. Name & R.A. & Decl. & $\begin{array}{l}\text { Opt. } \\
\text { Type }\end{array}$ & Class & $z$ & $S_{5}$ & $\begin{array}{l}\text { LAT } \\
\text { Flux }\end{array}$ & $\begin{array}{c}\Delta \text { LAT } \\
\text { Flux }\end{array}$ & Core $T_{B}$ & $\begin{array}{l}\text { Open. } \\
\text { Ang. }\end{array}$ & $\Delta$ P.A. \\
\hline F18525+4853 & 1FGL J1852.5+4853 & CGRaBS J1852+4855 & $18: 52: 28.550$ & $48: 55: 47.50$ & bzq & PS & 1.25 & 420 & 51.36 & 22.81 & $7.41 \mathrm{e}+11$ & $\cdots$ & $\ldots$ \\
\hline F19030+5539 & 1FGL J1903.0+5539 & CRATES J1903+5540 & 19:03:11.611 & $55: 40: 38.39$ & bzb & LJET & $\ldots$ & 180 & 37.53 & 15.16 & $2.49 \mathrm{e}+10$ & 35 & -4 \\
\hline F19416+7214 & 1FGL J1941.6+7214 & CRATES J1941+7221 & 19:41:26.981 & $72: 21: 42.19$ & agu & SJET & $\ldots$ & 157 & 66.46 & 29.70 & $2.18 \mathrm{e}+10$ & $\ldots$ & $\ldots$ \\
\hline F20000+6508 & 1FGL J2000.0+6508 & 1ES 1959+650 & 19:59:59.849 & $65: 08: 54.71$ & bzb & LJET & 0.049 & 213 & 71.92 & 8.60 & $2.31 \mathrm{e}+10$ & 39 & 2 \\
\hline F20019+7040 & 1FGL J2001.9+7040 & CRATES J2001+7040 & 20:01:33.950 & $70: 40: 25.82$ & agu & LJET & 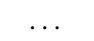 & 36.5 & 27.18 & 10.17 & $5.63 e+9$ & $\ldots$ & $\ldots$ \\
\hline F20060+7751 & 1FGL J2006.0+7751 & CGRaBS J2005+7752 & 20:05:31.001 & $77: 52: 43.21$ & bzb & LJET & 0.342 & 904 & 51.58 & 22.83 & $9.70 \mathrm{e}+10$ & $\ldots$ & $\ldots$ \\
\hline F20091+7228 & 1FGL J2009.1+7228 & $4 \mathrm{C}+72.28$ & 20:09:52.301 & $72: 29: 19.39$ & bzb & LJET & $\ldots$ & 775 & 46.83 & 13.78 & $1.62 \mathrm{e}+10$ & $\ldots$ & $\ldots$ \\
\hline F20204+7608 & 1FGL J2020.4+7608 & CGRaBS J2022+7611 & 20:22:35.590 & $76: 11: 26.20$ & bzb & LJET & $\ldots$ & 774 & 45.59 & 19.42 & $1.19 \mathrm{e}+11$ & 33 & 27 \\
\hline F20315+1219 & 1FGL J2031.5+1219 & PKS 2029+121 & 20:31:55.001 & $12: 19: 41.30$ & bzb & SJET & $1.215^{\mathrm{b}}$ & 1080 & 50.42 & 21.03 & $4.74 \mathrm{e}+11$ & $\ldots$ & $\ldots$ \\
\hline F20354+1100 & 1FGL J2035.4+1100 & PKS 2032+107 & 20:35:22.339 & $10: 56: 06.79$ & bzq & LJET & 0.601 & 522 & 74.93 & 14.31 & $1.36 \mathrm{e}+11$ & $\ldots$ & $\ldots$ \\
\hline F20497+1003 & 1FGL J2049.7+1003 & PKS 2047+098 & 20:49:45.859 & 10:03:14.40 & agu & LJET & $\ldots$ & 698 & 58.69 & 25.99 & $6.07 \mathrm{e}+10$ & $\ldots$ & $\ldots$ \\
\hline F21155+2937 & 1FGL J2115.5+2937 & B2 2113+29 & 21:15:29.419 & $29: 33: 38.41$ & bzq & LJET & 1.514 & 705 & 39.17 & 11.71 & $7.26 \mathrm{e}+9$ & $\ldots$ & $\ldots$ \\
\hline $\mathrm{F} 21161+3338$ & 1FGL J2116.1+3338 & B2 2114+33 & 21:16:14.520 & $33: 39: 20.41$ & bzb & LJET & $\ldots$ & 49.5 & 38.92 & 15.69 & $4.46 e+9$ & $\ldots$ & $\ldots$ \\
\hline F21209+1901 & 1FGL J2120.9+1901 & OX 131 & 21:21:00.610 & 19:01:28.31 & bzq & LJET & 2.18 & 363 & 43.68 & 17.31 & $2.25 \mathrm{e}+10$ & 21 & 5 \\
\hline F21434+1742 & 1FGL J2143.4+1742 & OX 169 & 21:43:35.539 & $17: 43: 48.68$ & bzq & SJET & 0.211 & 728 & 184.60 & 10.49 & $1.52 \mathrm{e}+11$ & $\ldots$ & $\ldots$ \\
\hline $\mathrm{F} 21525+1734$ & 1FGL J2152.5+1734 & PKS 2149+17 & $21: 52: 24.821$ & $17: 34: 37.81$ & bzb & SJET & 0.871 & 495 & 20.25 & 7.86 & $1.51 \mathrm{e}+10$ & $\ldots$ & $\ldots$ \\
\hline F21574+3129 & 1FGL J2157.4+3129 & B2 2155+31 & $21: 57: 28.819$ & $31: 27: 01.40$ & bzq & SJET & 1.486 & 479 & 53.99 & 10.54 & $3.57 \mathrm{e}+11$ & $\ldots$ & $\ldots$ \\
\hline F22035+1726 & 1FGL J2203.5+1726 & PKS 2201+171 & 22:03:26.890 & $17: 25: 48.29$ & bzq & LJET & 1.076 & 692 & 93.40 & 8.59 & $6.52 \mathrm{e}+10$ & $\ldots$ & $\ldots$ \\
\hline $\mathrm{F} 22121+2358$ & 1FGL J2212.1+2358 & PKS 2209+236 & 22:12:05.971 & $23: 55: 40.58$ & bzq & LJET & 1.125 & 902 & 21.37 & 8.40 & $1.30 \mathrm{e}+11$ & $\ldots$ & $\ldots$ \\
\hline $\mathrm{F} 22171+2423$ & 1FGL J2217.1+2423 & B2 2214+24B & 22:17:00.830 & $24: 21: 46.01$ & bzb & LJET & 0.505 & 550 & 42.39 & 8.32 & $3.30 \mathrm{e}+11$ & $\ldots$ & $\ldots$ \\
\hline F22193+1804 & 1FGL J2219.3+1804 & CGRaBS J2219+1806 & 22:19:14.090 & 18:06:35.60 & bzq & SJET & 1.071 & 169 & 25.49 & 10.40 & $4.80 \mathrm{e}+10$ & $\ldots$ & $\cdots$ \\
\hline $\mathrm{F} 22362+2828$ & 1FGL J2236.2+2828 & B2 2234+28A & 22:36:22.469 & $28: 28: 57.40$ & bzq & SJET & 0.795 & 1500 & 84.96 & 8.23 & $2.62 \mathrm{e}+11$ & $\ldots$ & $\ldots$ \\
\hline $\mathrm{F} 22440+2021$ & 1FGL J2244.0+2021 & CRATES J2243+2021 & 22:43:54.739 & $20: 21: 03.82$ & bzb & LJET & $\ldots$ & 60.5 & 38.41 & 14.31 & $1.51 \mathrm{e}+10$ & $\cdots$ & $\cdots$ \\
\hline $\mathrm{F} 22501+3825$ & 1FGL J2250.1+3825 & B3 $2247+381$ & 22:50:05.750 & $38: 24: 37.19$ & bzb & SJET & 0.119 & 56.5 & 26.04 & 10.72 & $5.33 e+9$ & $\cdots$ & $\cdots$ \\
\hline $\mathrm{F} 22517+4030$ & 1FGL J2251.7+4030 & CRATES J2251+4030 & 22:51:59.770 & $40: 30: 58.21$ & bzb & SJET & $\ldots$ & 63.2 & 46.51 & 19.73 & $9.07 e+9$ & $\ldots$ & $\ldots$ \\
\hline F22539+1608 & 1FGL J2253.9+1608 & 3C 454.3 & 22:53:57.751 & $16: 08: 53.59$ & bzq & LJET & 0.859 & 11200 & 1355.47 & 17.05 & $5.91 \mathrm{e}+11$ & 36 & 24 \\
\hline F23073+1452 & 1FGL J2307.3+1452 & CGRaBS J2307+1450 & 23:07:34.001 & 14:50:17.99 & bzb & LJET & $0.503^{\mathrm{b}}$ & 50.7 & 24.64 & 9.21 & $2.32 \mathrm{e}+10$ & $\ldots$ & $\ldots$ \\
\hline $\mathrm{F} 23110+3425$ & 1FGL J2311.0+3425 & B2 $2308+34$ & 23:11:05.330 & $34: 25: 10.88$ & bzq & LJET & 1.817 & 808 & 53.68 & 9.52 & $5.80 \mathrm{e}+10$ & $\ldots$ & $\ldots$ \\
\hline $\mathrm{F} 23220+3208$ & 1FGL J2322.0+3208 & B2 2319+31 & 23:21:54.950 & 32:04:07.61 & bzq & LJET & 1.489 & 475 & 40.48 & 17.44 & $2.32 \mathrm{e}+11$ & $\ldots$ & $\ldots$ \\
\hline F $23216+2726$ & 1FGL J2321.6+2726 & $4 \mathrm{C}+27.50$ & 23:21:59.861 & $27: 32: 46.39$ & bzq & LJET & 1.253 & 941 & 41.26 & 18.15 & $4.25 \mathrm{e}+10$ & $\ldots$ & $\ldots$ \\
\hline $\mathrm{F} 23226+3435$ & 1FGL J2322.6+3435 & CRATES J2322+3436 & 23:22:44.011 & $34: 36: 13.90$ & bzb & PS & 0.098 & 27.3 & 34.87 & 0.00 & $6.68 \mathrm{e}+9$ & $\ldots$ & $\ldots$ \\
\hline $\mathrm{F} 23252+3957$ & 1FGL J2325.2+3957 & B3 $2322+396$ & 23:25:17.870 & $39: 57: 36.50$ & bzb & SJET & $\ldots$ & 139 & 27.66 & 8.30 & $1.24 \mathrm{e}+10$ & $\ldots$ & $\ldots$ \\
\hline
\end{tabular}

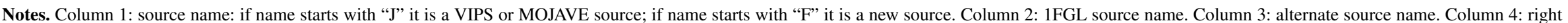

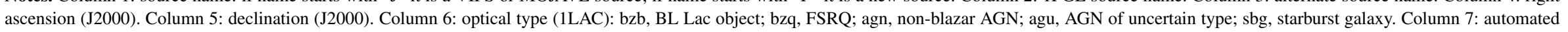

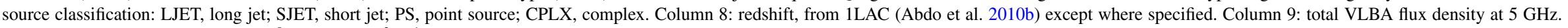

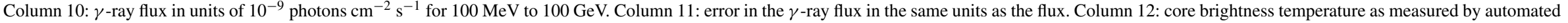
program (Helmboldt et al. 2007). Column 13: opening angle in degrees. Column 14: change in jet position angle in degrees.

${ }^{a}$ A LAT source which is associated with multiple radio sources with high $(\geqslant 80 \%)$ probability in 1LAC.

${ }^{\mathrm{b}}$ Redshift obtained from NED.

${ }^{\mathrm{c}}$ MOJAVE source. For more data, visit the MOJAVE Web site http://www.physics.purdue.edu/astro/MOJAVE/MOJAVEIItable.html or see Lister et al. (2009a). 
BL Lac object VIPS J12248+4335 (probability 94\%) and the FSRQ VIPS J12269+4340 (probability 87\%). The LAT source 1FGL J0448.6+1118 is associated with the FSRQ CRATES J0448+1127 (F04486+112A in our sample; probability 99\%) and the BL Lac object PKS 0446+11 (F04486+112B in our sample; probability $92 \%$ ). The LAT source 1FGL J0510.0+1800 is associated with an AGN of unknown type CRATES J0509+1806 (F05100+180A; probability 91\%) and the FSRQ PKS 0507+17 (F05100+180B; probability 100\%). It is possible that all of these sources are emitting $\gamma$-rays, so we included all of them in our calculations. Where possible, we use the 1LAC redshifts. If the source did not have a redshift listed in 1LAC, we searched the NASA/IPAC Extragalactic Database (NED).

To build a non-LAT-detected sample for comparison, we excluded all LAT sources from VIPS, leaving 1018 objects in our non-LAT sample. Of these 1018 objects, 24 are BL Lac objects, 479 are FSRQs, and 515 are AGN/Other types (radio galaxies or AGNs of uncertain type). The optical types for VIPS sources were adopted from the Candidate Gamma-Ray Blazar Survey (CGRaBS; Healey et al. 2008).

\section{VLBA DATA REDUCTION}

All VLBA data were correlated using the new DiFX software correlator (e.g., Deller et al. 2011). The correlated data were processed via the VIPS data reduction pipeline using automated scripts. See Taylor et al. (2005) and Helmboldt et al. (2007) for a more thorough description of the VIPS pipeline. Initial calibration (fringe-fitting, flux and phase calibration, polarization calibration) was done using the Astronomical Image Processing System (AIPS; Greisen 2003). Imaging and visibility model fitting were done using Difmap (Shepherd 1997).

\section{GAMMA-RAY FLUX AND RADIO FLUX DENSITY}

Throughout the rest of this paper, we will use the nonparametric Spearman test (e.g., Press et al. 1986) to look for correlations between the LAT-detected and non-LAT-detected objects. The Spearman correlation coefficient $\left(\rho_{s}\right)$ has a range of $0<\left|\rho_{s}\right|<1$. A high value of $\rho_{s}$ indicates a significant correlation. This is a powerful test for statistical correlation, but it does not test an actual physical correlation. In order to be certain our correlations are physically significant, we ensure that a redshift selection effect is not adding a bias to our data.

The LAT measures $\gamma$-ray flux in several bands. To create total $\gamma$-ray fluxes, we combined the fluxes from three bands: 100-300 MeV, $300 \mathrm{MeV}-1 \mathrm{GeV}$, and 1-100 GeV. The fluxes were added and uncertainties were added in quadrature. However, some sources had only upper limits to their fluxes in certain bands. If a source's reported fluxes in one or two bands were upper limits, we use $1 / 2$ the reported flux as the uncertainty in that band as the upper limits are given as $2 \sigma$ results (Abdo et al. 2010a). If a source had upper limits on its flux in all three bands, we adopt the convention of listing its error as 0.00 .

\subsection{Redshift Selection Effect}

Imagine a population of sources covering a wide range of redshifts in which the radio and $\gamma$-ray emission is not physically correlated, but in which radio flux and $\gamma$-ray flux are correlated with redshift. This is actually what one would naively expect because the more distant objects will be fainter. Such a population will show a strong correlation of radio flux with gamma-ray flux via the Spearman test, but this does not

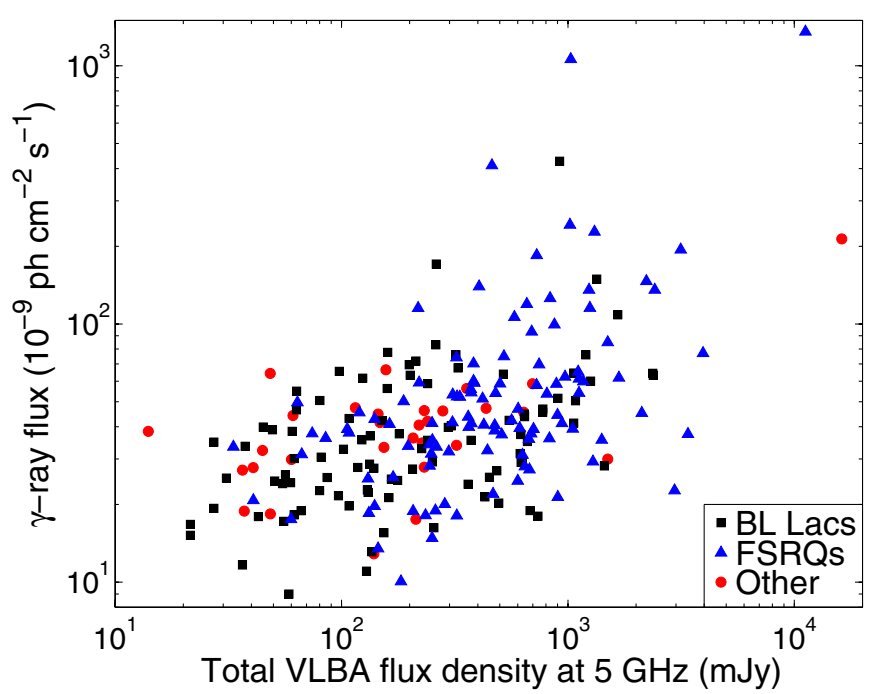

Figure 1. LAT $\gamma$-ray flux $(100 \mathrm{MeV}-100 \mathrm{GeV})$ vs. total VLBA radio flux density at $5 \mathrm{GHz}$. The $\gamma$-ray fluxes are in units of $10^{-9}$ photons $\mathrm{cm}^{-2} \mathrm{~s}^{-1}$. The black squares are BL Lac objects, the blue triangles are FSRQs, and the red circles are radio galaxies and unclassified objects. Error bars are omitted for ease of viewing.

indicate a significant physical correlation between these two observables.

However, by investigating the relationship between radio flux density at $5 \mathrm{GHz}\left(S_{5}\right)$ and redshift for the LAT-detected sources we can rule out a correlation between the two. We calculated the Spearman $\rho_{s}$ values for the $S_{5}-z$ relationship for the BL Lac objects and FSRQs separately. For the BL Lac objects, the $\rho_{s}$ value was 0.308 , with a $2.1 \%$ probability that random sampling would produce this same $\rho_{s}$ value. For the FSRQs, the $\rho_{s}$ value was 0.109 , with a $26.2 \%$ probability of getting the same value by random sampling. Therefore, there is a very marginal correlation between the radio flux density and redshift for our BL Lac objects, and no correlation for our FSRQs.

We also tested the correlation between $\gamma$-ray flux and redshift. The $\rho_{s}$ values were 0.084 for BL Lac objects and 0.016 for FSRQs, with the probability of getting the same values from random sampling of $54.0 \%$ and $86.8 \%$, respectively. So, there is no significant correlation between $\gamma$-ray flux and redshift for either BL Lac objects or FSRQs. We also visually inspected plots of both LAT flux and total VLBA radio flux density versus redshift and saw no obvious trends, confirming the correlation coefficient results.

\subsection{Gamma-Ray Flux versus Radio Flux Density}

In Figure 1, we plot the LAT flux versus the total VLBA flux density at $5 \mathrm{GHz}$. Again, the LAT fluxes are broadband fluxes from $100 \mathrm{MeV}$ to $100 \mathrm{GeV}$.

The Spearman $\rho_{s}$ values we found were 0.467 for the BL Lac objects and 0.510 for the FSRQs. The probabilities for getting the same $\rho_{s}$ values from random sampling were $2 \times 10^{-6}$ for the BL Lac objects and $2 \times 10^{-8}$ for the FSRQs. Therefore, the LAT flux correlates very strongly with the total VLBA flux density. The BL Lac objects do not correlate quite as strongly, especially considering that we found a marginal correlation between total VLBA flux density and redshift for these objects in the previous section. Still, this provides solid evidence that objects with higher radio flux density also produce more $\gamma$-ray flux. 

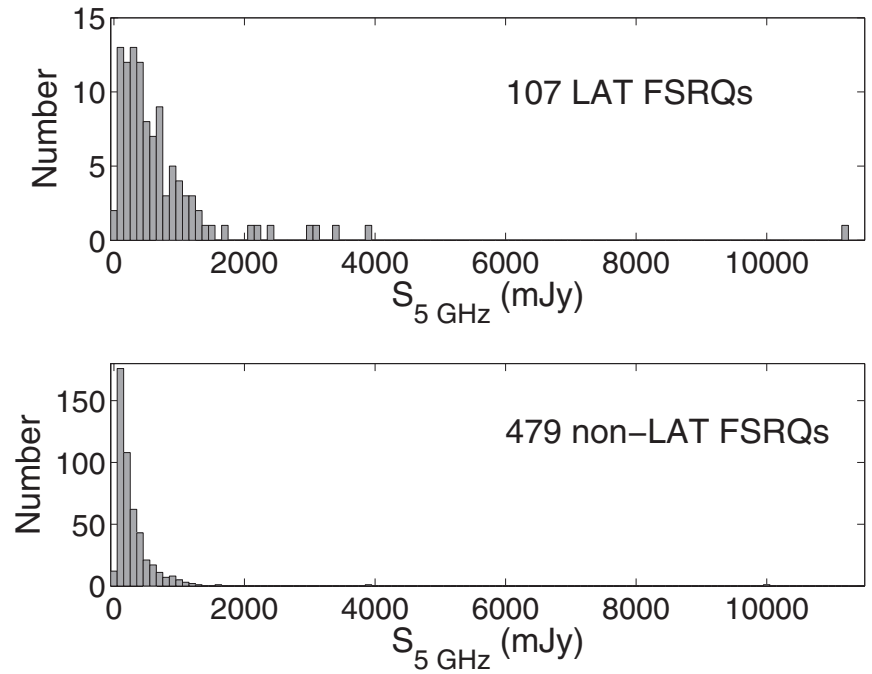

Figure 2. Distributions of LAT (top) and non-LAT (bottom) FSRQ total VLBA flux density at $5 \mathrm{GHz}$.

In our previous paper (Linford et al. 2011), we found that there was no strong correlation between radio flux density and $\gamma$-ray flux. However, our flux densities for those observations were prior to the launch of Fermi. Furthermore, it is likely that the radio flux density increases during episodes of $\gamma$-ray flaring (e.g., Kovalev et al. 2009). Our newer, larger, and more contemporaneous set of observations are more appropriate for making these comparisons.

\subsection{Radio Flux Density}

The median total VLBA flux density at $5 \mathrm{GHz}$ was $177 \mathrm{mJy}$ for the LAT BL Lac objects and $221 \mathrm{mJy}$ for the non-LAT BL Lac objects. The LAT FSRQ appeared to have significantly higher flux densities than the non-LAT FSRQ, with a median of $467 \mathrm{mJy}$ for LAT FSRQs compared to a median of only $191 \mathrm{mJy}$ for non-LAT FSRQs. The LAT AGN/Other objects also had higher $5 \mathrm{GHz}$ flux densities than their non-LAT source counterparts. The Kolmogorov-Smirnov (K-S) test probability that the LAT and non-LAT BL Lac objects belong to the same parent population is about $38 \%$. The $\mathrm{K}-\mathrm{S}$ test probability that the LAT and non-LAT FSRQs are related is only $3 \times 10^{-12}$. See Figure 2 for a plot of the FSRQ total VLBA $5 \mathrm{GHz}$ flux density distributions.

\section{ARCHIVAL VERSUS CONTEMPORANEOUS}

In this section we investigate the changes in the 90 sources that were part of VIPS (i.e., observed in 2006 or earlier) and our new sample (observed in 2009-2010). Of these 90 sources, 35 are BL Lac objects, 44 are FSRQs, and 11 are AGN/Other objects. It is important to note that we do not know whether most of our sources were emitting $\gamma$-rays in the archival data. It is possible that many of them would have been detected by Fermi had it been in operation at the time. Only six of these sources were detected by the Energetic Gamma-Ray Experiment Telescope (EGRET) on board the Compton Gamma Ray Observatory satellite. As an estimate of the uncertainty of the median values we will use the median absolute deviation (MAD) given by

$$
\operatorname{MAD}(X)=\operatorname{median}\left(\mid\left(X_{i}-\operatorname{median}(X) \mid\right),\right.
$$

where $X_{i}$ is a value in the array $X$. In other words, it is the median of the positive difference between each entry in $X$ and the overall median of $X$. See Huber \& Ronchetti (2009) for more information on the MAD.

\subsection{Radio Flux Density Variability}

It is well known that blazars are highly variable sources in the radio and $\gamma$-ray regimes. Several studies have also indicated that the radio and $\gamma$-ray variability are correlated (e.g., Kovalev et al. 2009 and Schinzel et al. 2010). Looking at the differences in total VLBA radio flux densities (see Figure 3) for 90 of our sources that were observed both in 2006 (or earlier) and in 2009 , we saw that while there is variability the magnitude of the variability does not appear to correlate with the $\gamma$-ray flux. The median total flux densities showed little significant change between the two epochs. The BL Lac objects had a median flux density of $149.2 \mathrm{mJy}$ in the archival data and $133.2 \mathrm{mJy}$ in the new data. The difference between the medians for the two epochs falls well within the median absolute deviations of $74 \mathrm{mJy}$ for archival data and $72 \mathrm{mJy}$ for the current data. The FSRQs appeared to become somewhat brighter in the new data, with a median flux density of $256.2 \mathrm{mJy}$ in the archival data and $316.0 \mathrm{mJy}$ in the new data. Again, the difference between the two epochs is not significant when compared to the median absolute deviations of $122 \mathrm{mJy}$ for the archival data and $176 \mathrm{mJy}$ for the current data.

We also looked at the radio emission from the core (the bright compact component at the base of the jet). The core flux density was found using automatic visibility fitting (see Taylor et al. 2007 for a more detailed discussion of this process). See Figure 4 for a plot of the difference in core flux density (current minus archival) versus the total LAT flux. Again, we found that there is no apparent correlation between core flux density variability and LAT flux. The median core flux for the FSRQs is higher during $\gamma$-ray detection, going from $225.1 \mathrm{mJy}$ in the archival data to $293.6 \mathrm{mJy}$ in the new data, but this is again within the median absolute deviations of $107 \mathrm{mJy}$ for the archival data and $156 \mathrm{mJy}$ for the new data. Also, from Figure 4 we can see that most of the FSRQs are actually slightly dimmer, with 25 of the 44 FSRQs showing a reduction in core flux density.

\subsection{Core Brightness Temperature Variability}

We obtained the brightness temperatures from automatic model-fitting procedure by fitting to the visibility data directly (Taylor et al. 2007). The minimum observable size for each source was calculated using Equation (2) from Kovalev et al. (2005), where we computed the signal-to-noise ratio of each core using the core flux density, the rms measured from the $5 \mathrm{GHz}$ image, and a beam FWHM of 3 milliarcseconds (mas), the largest dimension of our restoring beam. For those sources where the estimated core size was less than this minimum size, we used the minimum size to compute the brightness temperature.

The core brightness temperatures are split almost evenly between those that are lower than they were prior to LAT detection and those that are higher. Nearly all had core brightness temperatures in 2009 that were either within $5 \%$ of the archival value or higher than the archival value. The median core brightness temperature for the BL Lac objects was $2.3 \times 10^{10} \mathrm{~K}$ for the archival data and $2.9 \times 10^{10} \mathrm{~K}$ for the new data. The median core brightness temperature was $8.2 \times 10^{10} \mathrm{~K}$ for the FSRQs in the archival data and $6.4 \times 10^{10} \mathrm{~K}$ for the new data. However, the median absolute deviations were $6.34 \times 10^{10}$ for the archival data and $5.13 \times 10^{10}$ for the new data, so 

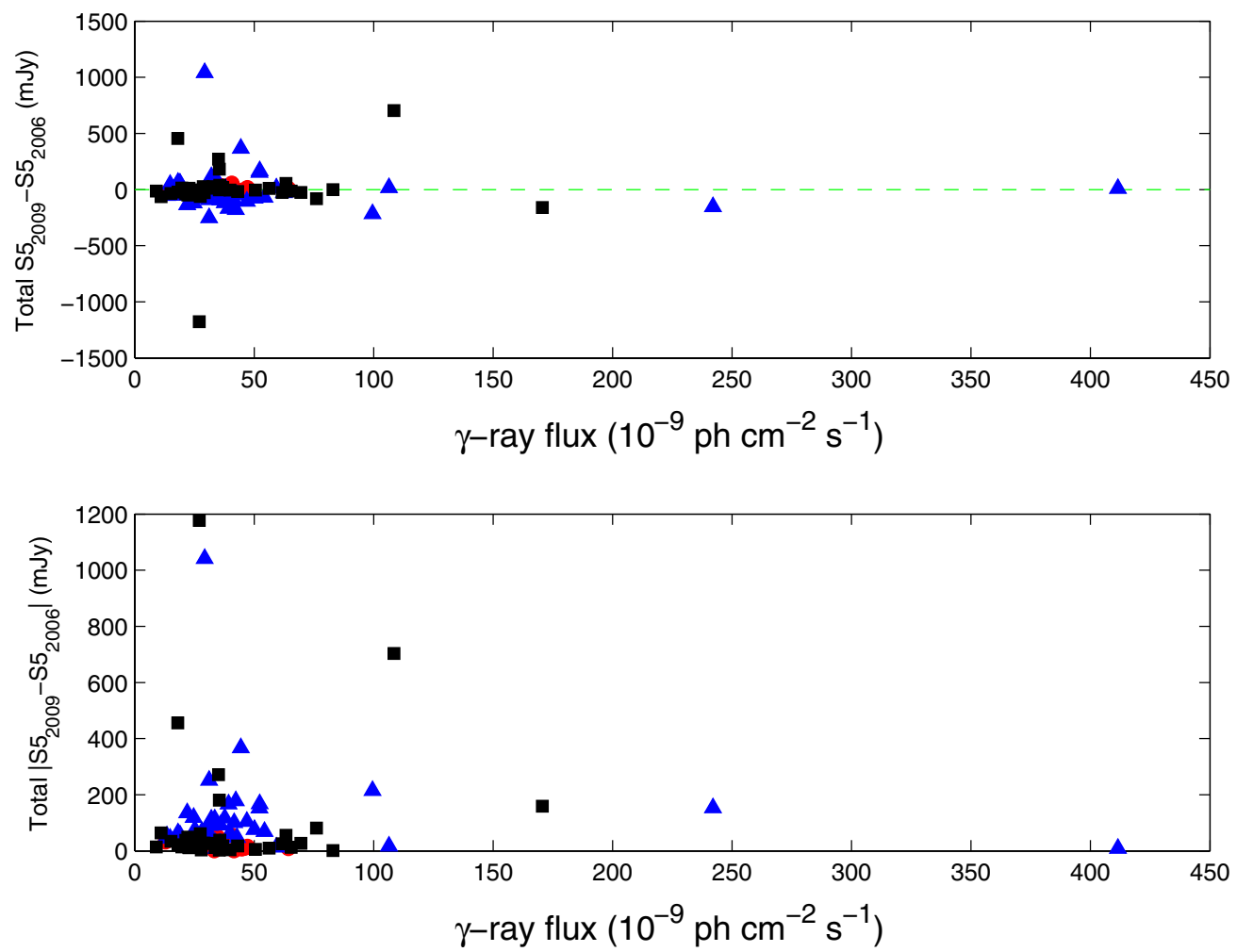

Figure 3. Top: difference in total flux density at $5 \mathrm{GHz}$ (current - archival) vs. total LAT flux. Bottom: absolute value of the difference in total flux density (current - archival) vs. total LAT flux. Black squares are BL Lac objects, blue triangles are FSRQs, and red circles are AGN/Other.
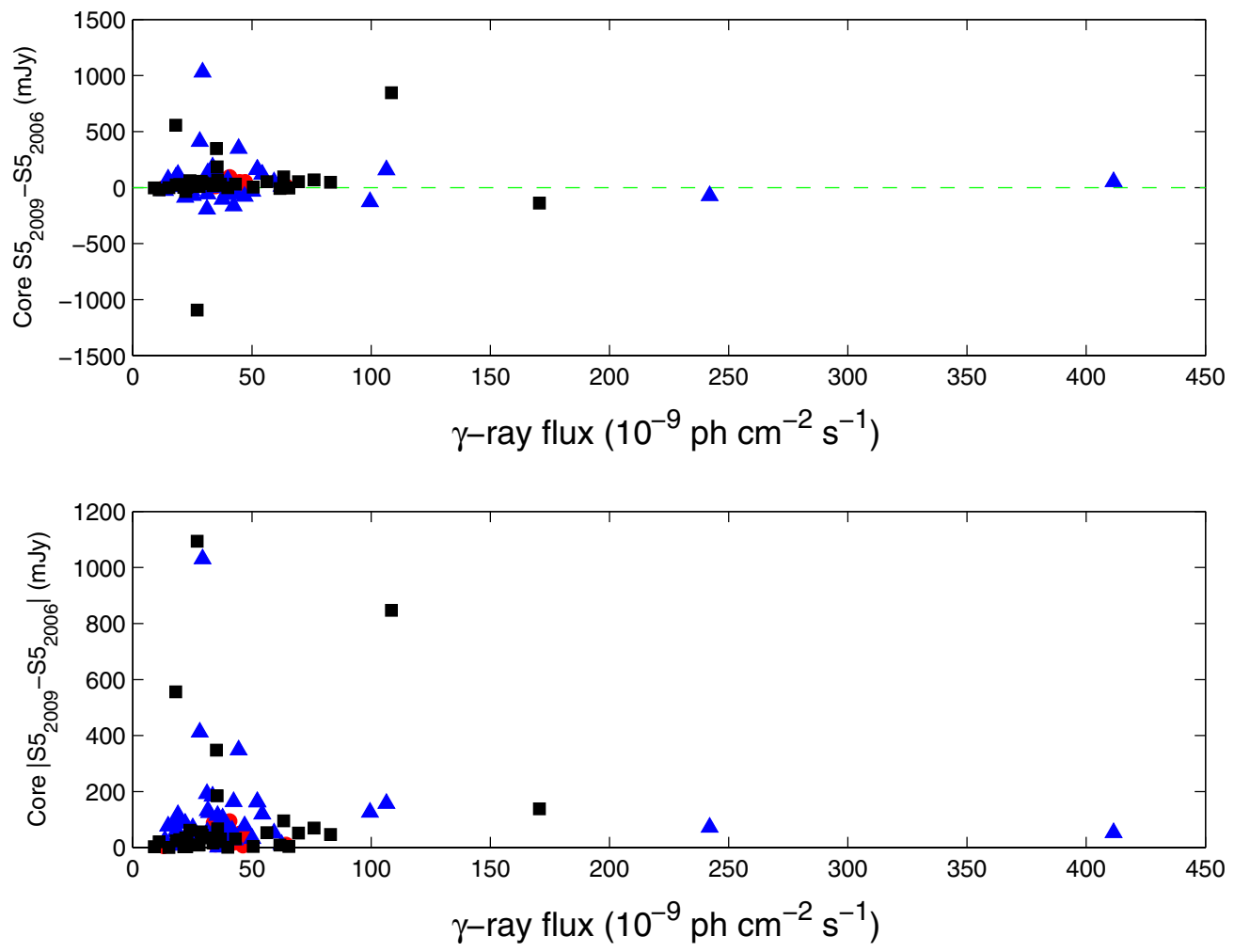

Figure 4. Top: difference in core flux density at $5 \mathrm{GHz}$ (current - archival) vs. total LAT flux. Bottom: absolute value of the difference in core flux density (current archival) vs. total LAT flux. Black squares are BL Lac objects, blue triangles are FSRQs, and red circles are AGN/Other. 


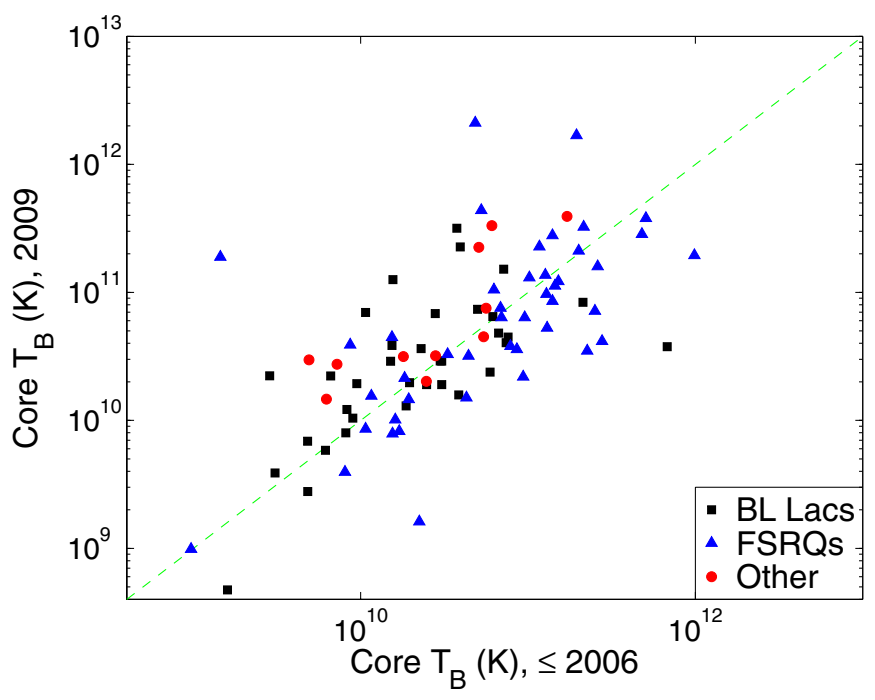

Figure 5. Core brightness temperatures, current data (2009-2010) vs. archival data (prior to or during 2006).

the difference in the medians is not a significant change. See Figure 5 for a plot of the core flux densities in both epochs.

\subsection{Core Polarization Variability}

To measure the polarization properties of our sources, we used the Gaussian mask method described by Helmboldt et al. (2007). In order to be considered a "polarized" source, the source had to have a polarized flux of at least $0.3 \%$ of the Stokes $I$ peak value (to avoid leakage contamination) and have at least a $5 \sigma$ detection (compared to the noise image generated by the AIPS task COMB). We obtained measurements of core, jet, and total fractional polarization. Of these, only the core fractional polarization yielded interesting results. We detected polarization in the cores of $80 \%$ ( 72 of 90 ) of the sources for which we had observations in two epochs. Of our 90 sources, 48 showed higher core fractional polarization in the new data than the archival data. This includes 17 BL Lac objects, 28 FSRQs, and 3 AGN/Other objects. Only 15 sources had no polarized flux in their cores in both epochs. Twenty-seven of our sources showed a decrease in core fractional polarization in the new data including $11 \mathrm{BL} \mathrm{Lac}$ objects, 12 FSRQs, and 4 AGN/Other objects. Of the objects that showed a decrease in core fractional polarization, only three (one BL Lac object and two FSRQs) were detected as polarized in the archival data and had no polarized flux in the core in the new data. See Figure 6 for a plot showing how the core fractional polarizations have changed between the two epochs.

\section{LAT DETECTED VERSUS NON-LAT DETECTED}

Throughout this section we compare our large, contemporaneous sample with the non-LAT-detected sources in VIPS.

\subsection{Source Classes}

We used the automatic classification script from Helmboldt et al. (2007) to classify our sources by appearance for comparison with the VIPS sample. We also went through them by eye and reclassified any objects that the script misidentified. Sources were classified as point sources (PS), short jets (SJET), long jets (LJET), complex (CPLX), or compact symmetric object candidates (CSO). LJET means the source has a jet with an angular extent of at least 6 mas. SJET means the source has a

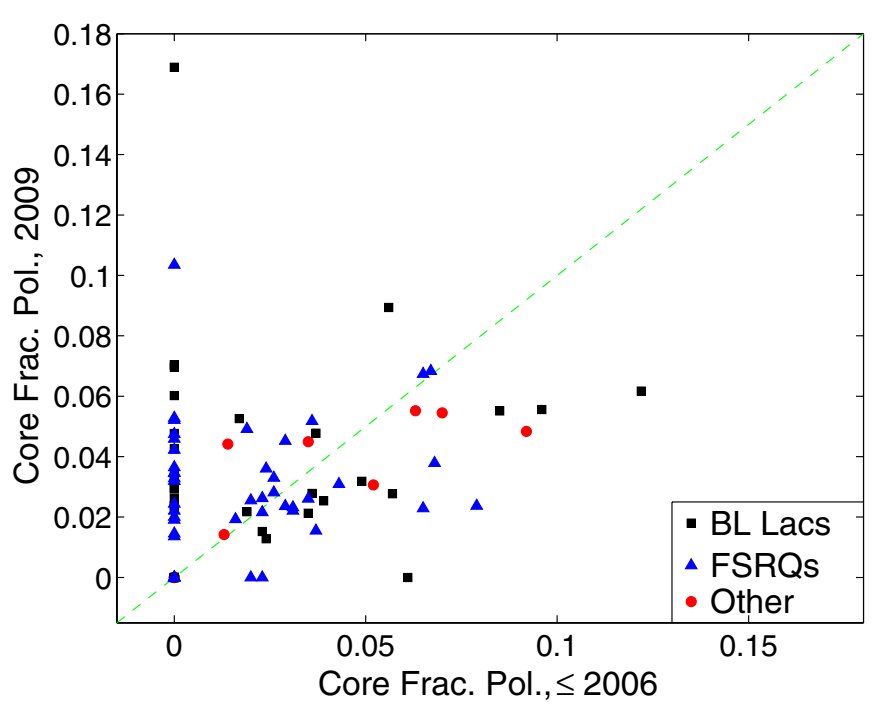

Figure 6. Core fractional polarization at $5 \mathrm{GHz}$, current data (2009-2010) vs. archival data (prior to or during 2006).

discernible jet, but with an angular extent less than 6 mas. PS means the source has no discernible jet. CPLX indicates that the source has complicated structure beyond the typical point source or core-jet morphology. See Table 2 for a breakdown of the source classifications for both LAT and non-LAT sources. From this table, it appears that $\gamma$-ray bright blazars are more likely to be LJET than the blazars not detected by the LAT. Also, note the lack of CSO candidate objects among the $\gamma$-ray bright population. This is something we also reported in Linford et al. (2011).

\subsection{Core Brightness Temperature}

As discussed in Section 5.2, we obtained the core brightness temperatures by automatic model fitting to the visibility data. The LAT and non-LAT BL Lac objects were similar, with a median core brightness temperature of $2.89 \times 10^{10} \mathrm{~K}$ for the LAT BL Lac objects and $2.65 \times 10^{10} \mathrm{~K}$ for the non-LAT. The FSRQs were quite different. The median core brightness temperatures were $6.36 \times 10^{10} \mathrm{~K}$ for the LAT FSRQs and $2.54 \times$ $10^{10} \mathrm{~K}$ for the non-LAT FSRQs. The K-S tests show that the LAT FSRQs are indeed very different from the non-LAT FSRQs, with only a $6 \times 10^{-8}$ chance that the two distributions are drawn from the same parent population. These results confirm those reported in Linford et al. (2011). See Figure 7 for a plot of the core brightness temperature distributions of LAT and non-LAT FSRQs.

\subsection{Jet Brightness Temperature}

Unlike the core brightness temperatures, the jet brightness temperatures (formally, the brightness temperature of the brightest jet component) were obtained by automatic model fitting in the image data. This was done because automatic visibility fitting to jet components has a tendency to go awry for complicated sources. As with the core brightness temperatures, the LAT BL Lac objects were similar to the non-LAT BL Lac objects but the FSRQs appeared to be somewhat different. The K-S test for the FSRQ jet brightness temperature distributions gave probability of $1 \times 10^{-5}$ that the two were drawn from the same parent population. See Figure 8 for the distributions of LAT and non-LAT FSRQ jet brightness temperatures. The medians for the FSRQs with measured jet brightness temperatures were 
Table 2

Source Classifications

\begin{tabular}{|c|c|c|c|c|c|c|c|}
\hline LAT/non-LAT & Opt Type & LJET & SJET & PS & CPLX & CSO & Unidentified \\
\hline \multicolumn{8}{|l|}{ LAT-detected } \\
\hline & BL Lac objects & $55(58 \%)$ & $25(26 \%)$ & $12(13 \%)$ & $3(3 \%)$ & $\ldots$ & $\ldots$ \\
\hline & FSRQs & $54(50 \%)$ & $30(28 \%)$ & $21(20 \%)$ & $2(2 \%)$ & $\ldots$ & $\ldots$ \\
\hline & AGN/Other & $21(70 \%)$ & $5(17 \%)$ & $4(13 \%)$ & $\ldots$ & $\ldots$ & $\ldots$ \\
\hline \multicolumn{8}{|c|}{ Non-LAT-detected } \\
\hline & BL Lac objects & $11(46 \%)$ & $7(29 \%)$ & $6(25 \%)$ & $\ldots$ & $\ldots$ & $\ldots$ \\
\hline & FSRQs & $188(39 \%)$ & $121(25 \%)$ & $136(28 \%)$ & $2(\sim 1 \%)$ & $30(6 \%)$ & $2(\sim 1 \%)$ \\
\hline & AGN/Other & $214(42 \%)$ & $98(19 \%)$ & $111(21 \%)$ & $11(2 \%)$ & $71(14 \%)$ & $10(2 \%)$ \\
\hline
\end{tabular}

Notes. LJET: long jet; SJET: short jet; PS: point source; CPLX: complex; CSO: compact symmetric object candidate.
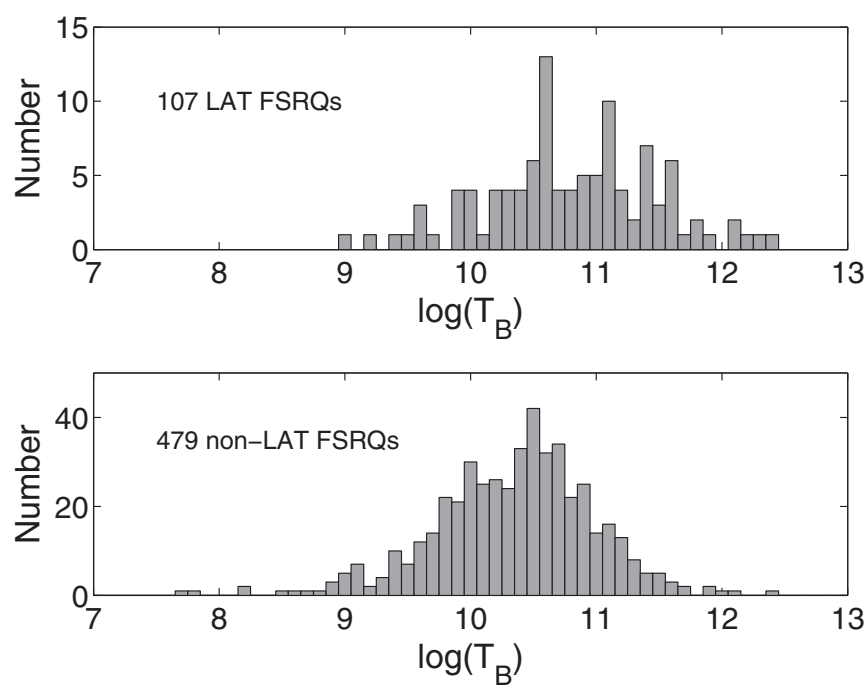

Figure 7. Distributions of core brightness temperatures for LAT-detected FSRQs (top) compared to the distributions of core brightness temperatures for non-LAT FSRQs in VIPS (bottom).

$9.8 \times 10^{7} \mathrm{~K}$ for LAT sources and $4.5 \times 10^{7} \mathrm{~K}$ for non-LAT sources.

\subsection{Jet Opening Angle, Bending, and Length}

We measured the mean apparent opening half-angle for each source with core-jet morphology. We used the following procedure (Taylor et al. 2007): we measured the separation of each jet component from the core along the jet axis (taken to be a linear fit to the component positions) and the distance of each component from the jet axis, i.e., $x^{\prime}$ and $y^{\prime}$ positions in a rotated coordinate system with the jet axis along the $x^{\prime}$-axis. For each component, we measured its extent from its center along a line perpendicular to the jet axis using the parameters of its elliptical fit, and then deconvolved this using the extent of the Gaussian restoring beam along the same line. The opening half-angle measured from each component is then taken to be

$$
\psi=\arctan \left[\left(\left|y^{\prime}\right|+d r\right) /\left|x^{\prime}\right|\right],
$$

where $d r$ is the deconvolved Gaussian size perpendicular to the jet axis. After measuring this for each jet component, we averaged them to get a single value. This was only done for sources with more than two total components, (i.e., at least two jet components).

The K-S tests for the LAT and non-LAT distributions found no significant differences between the two for any of the
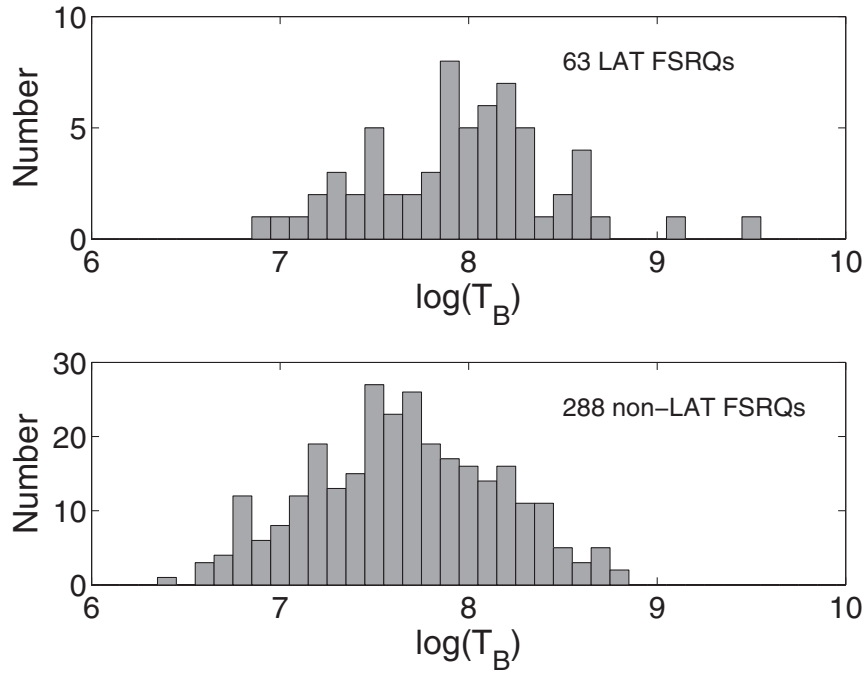

Figure 8. Distributions for jet brightness temperatures for LAT (top) and nonLAT (bottom) FSRQs.
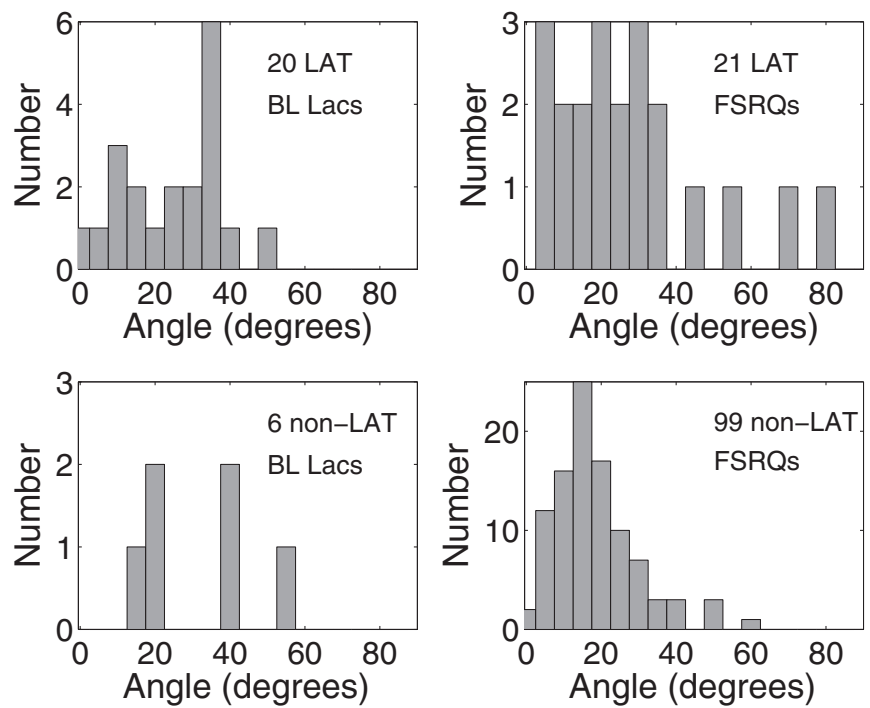

Figure 9. Distributions of apparent opening angles for BL Lac objects (left) and FSRQs (right) for both the LAT-detected (top) and non-LAT-detected (bottom) sources.

optical types. However, we noticed that our LAT sources have large apparent opening angles $\left(\geqslant 30^{\circ}\right)$ more often than the non-LAT sources, $37 \%$ of LAT sources versus $28 \%$ of non-LAT sources. See Figure 9 for plots of the opening angle distributions. Pushkarev et al. (2009) also reported that 

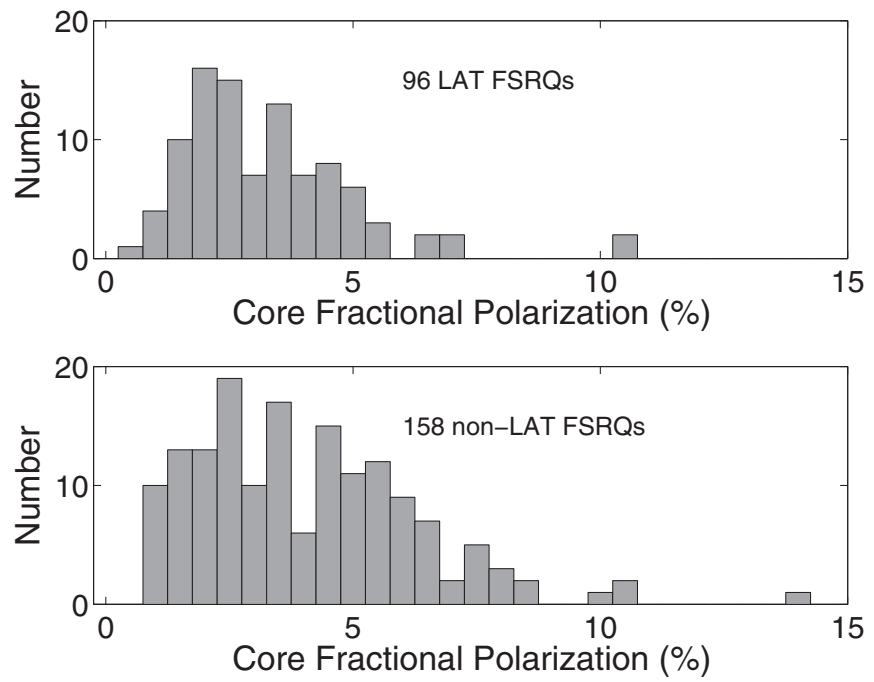

Figure 10. Distribution of core fractional polarization for LAT (top) and nonLAT (bottom) FSRQs.

LAT-detected MOJAVE sources in the 3 month LAT catalog tended to have larger opening angles than non-LAT sources. Ojha et al. (2010b) reported a tentative correlation between opening angle and $\gamma$-ray flux. We did not find any strong evidence of this. Using the Spearman test on all 49 of our sources with measured opening angles, we got a $\rho_{s}$ value of 0.2 and probability of getting the same $\rho_{s}$ value from random sampling of $16 \%$. Looking at the FSRQs alone, we found a $\rho_{s}$ of 0.5 and a $1.4 \%$ probability of getting the same result from random sampling. Therefore, there may be a marginally significant correlation between $\gamma$-ray flux and opening angles for FSRQs. Also, the K-S test result for the LAT and non-LAT FSRQs gave a $6.4 \%$ chance that the two distributions are drawn from the same parent sample. The BL Lac objects showed no significant correlation and no significant difference between the LAT and non-LAT distributions.

In order to make a larger sample for the K-S test, we combined the BL Lac objects and FSRQs into one sample. The result was a marginally significant difference between the LAT and non-LAT sources. The K-S test result was a $0.4 \%$ chance that the two distributions were drawn from the same parent population. We also applied the Spearman test on this sample to look for correlation with LAT flux. We did not find significant correlation, with a $\rho_{s}$ value of 0.3 and a probability of getting the same $\rho_{s}$ value from random sampling of $9 \%$.

Lister et al. (2011) reported a nonlinear correlation between $\gamma$-ray loudness (the ratio of $\gamma$-ray luminosity to radio luminosity) and apparent opening angle. They also found that all 19 of their sources with the large $\left(>40^{\circ}\right)$ apparent opening angles had a $\gamma$-ray loudness of more than 100 . They did not find a significant difference in the opening angle distributions between BL Lac objects and FSRQs. We are currently investigating these quantities in our data and we will report our results as soon as possible.

We also measure the change in jet position angle ("jet bending," see Helmboldt et al. 2008) and the length of the jet (the maximum separation between the core component and the jet components). We applied the K-S test to each of these measurements to see if the LAT and non-LAT distributions were different. We did not find any significant differences for either of these properties.

\subsection{Polarization}

We measured the polarization characteristics of our sources the same way as described in Section 5.3. As discussed therein, a source is considered to be polarized if it has a polarized flux of at least $0.3 \%$ of the Stokes $I$ peak and at least a $5 \sigma$ detection. For the LAT sources, 176 of 232 (about 76\%) showed core polarization. The FSRQs had the highest percentage of polarized sources with 96 of 107 (90\%). The BL Lac objects had 67 of 95 (71\%) sources with core polarization, and the AGN/Other sources had 13 of $30(43 \%)$ sources with core polarization. Compare these numbers with the non-LAT VIPS sources where only 270 of $1018(26.5 \%)$ had core polarization. For the non-LAT sample, the BL Lac objects were polarized most often, but only 10 of 24 (42\%). The non-LAT FSRQs had 158 out of 479 (33\%) sources with core polarization, and the AGN/Other only had 102 of 515 (20\%).

Despite the LAT sources having polarized cores more often, they do not appear to be more strongly polarized. The median core fractional polarization is $3.7 \%$ for LAT BL Lac objects and $3.1 \%$ for LAT FSRQs. The non-LAT sample has higher medians, with $4.9 \%$ for BL Lac objects and $3.7 \%$ for FSRQs. Overall, the median core fractional polarization for the LAT sample is $3.3 \%$ while the median for the non-LAT sample is $4.4 \%$. The K-S test showed no significant differences between the LAT and non-LAT BL Lac or AGN/Other objects. There is a marginally significant result for the FSRQs, with a probability of $0.2 \%$ that the LAT and non-LAT FSRQs were drawn from the same parent population. We plot the distribution of core fractional polarization for the FSRQs in Figure 10.

\section{NOTES ON INDIVIDUAL SOURCES}

In this section we present information on low-redshift $(z<$ 0.02 ) sources and non-blazar AGNs in our sample. Contour maps of these sources are shown in Figure 11, except for F03197+4130 (a.k.a. 3C84) as it is a very well known source.

\subsection{Low-redshift Objects}

Our sample contains three objects with redshifts of $z<0.02$.

F03197+4130, 1FGL J0319.7+4130, class LJET. This wellknown radio galaxy, 3C84, has a redshift of $z=0.018$ (1LAC). Its LAT flux was $213.59 \pm 10.69 \times 10^{-9}$ photons $\mathrm{cm}^{-2} \mathrm{~s}^{-1}$. We measured a total VLBA flux density of $16.2 \mathrm{Jy}$. Its core brightness temperature was $1.23 \times 10^{11} \mathrm{~K}$, which is relatively high for the AGN/Other objects. We did not detect any polarized flux from this object. This lack of strong polarization is well known and attributed to a Faraday screen consisting of the ionized gas which also produces the $\mathrm{H} \alpha$ in the Perseus cluster (Taylor et al. 2006). It has a jet and counterjet aligned north-south, with the northern jet showing significant free-free absorption (Walker et al. 2000). We measured the southern jet to be about 15.8 mas long. The northern counterjet had one dim component located about 11.8 mas from the core. No contour map for this source is provided here as it is a very famous source.

F09565+6938, 1FGL J0956.5+6938, class PS. With a redshift of $z=0.000677$ (de Vaucouleurs et al. 1991), the starburst galaxy M82 is one of two starburst galaxies in the 1FGL. It had a LAT flux of $38.40 \pm 16.23 \times 10^{-9}$ photons $\mathrm{cm}^{-2} \mathrm{~s}^{-1}$. We found a total VLBA flux density of $14 \mathrm{mJy}$. Its core brightness temperature was a relatively low $3.39 \times 10^{9} \mathrm{~K}$. We did not detect any polarization for this object. In the optical, this galaxy has a very striking $\mathrm{x}$-shape with bright filaments running perpendicularly through a spiral disk (e.g., Mutchler et al. 

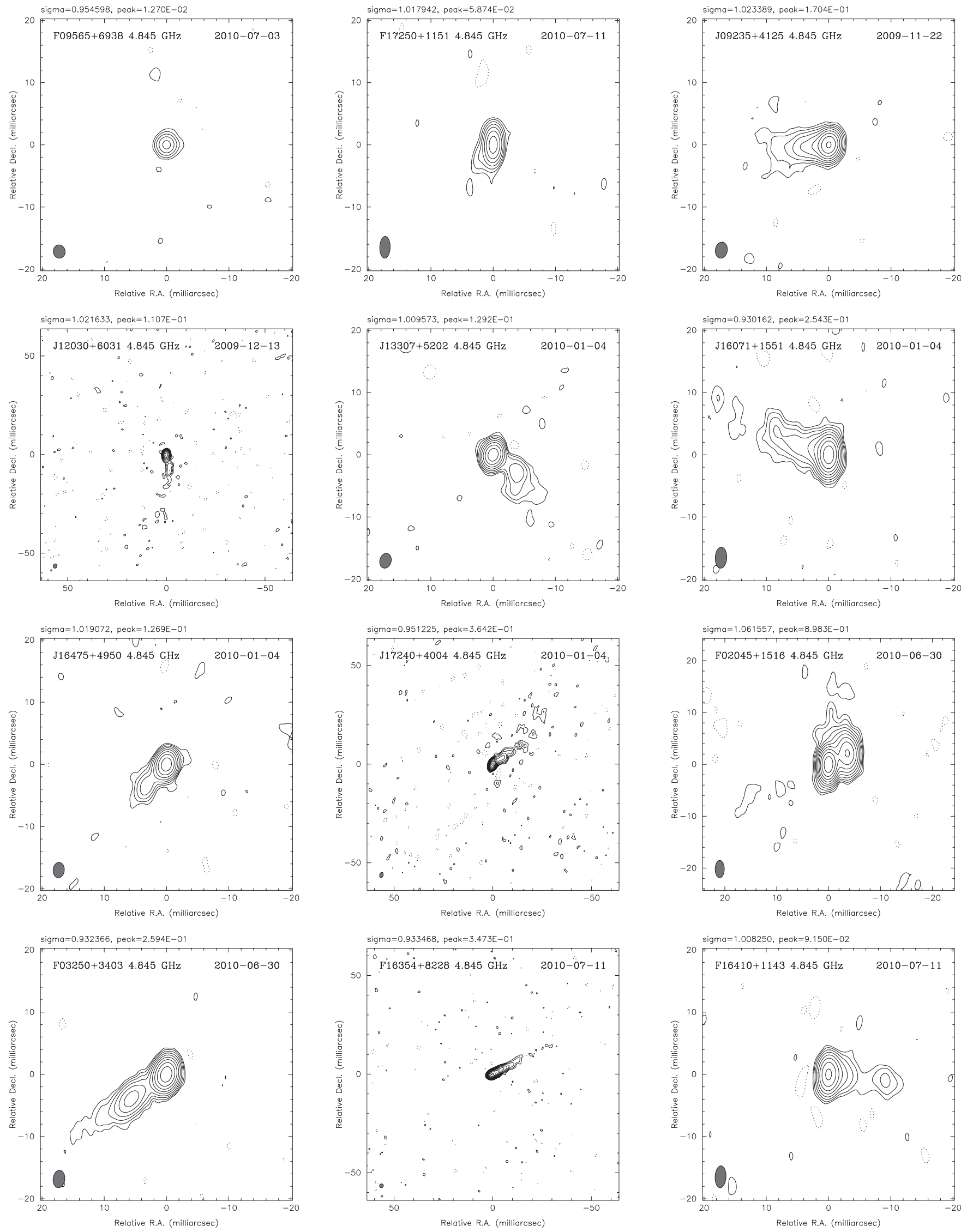

(a)

Figure 11. (a-c) Contour maps of sources in Section 7. All data were taken with the VLBA at $5 \mathrm{GHz}$. Bottom contours are all at $0.6 \mathrm{mJy}^{-1}$ beam ${ }^{-1}$, except for F02045+1516 which has a bottom contour of $1 \mathrm{mJy} \mathrm{beam}^{-1}$ (a) and except for F13060+7852 and F13213+8310 which have bottom contours of $0.8 \mathrm{mJy}$ beam ${ }^{-1}$ (c). The restoring beam is shown as an ellipse in the lower left corner. 

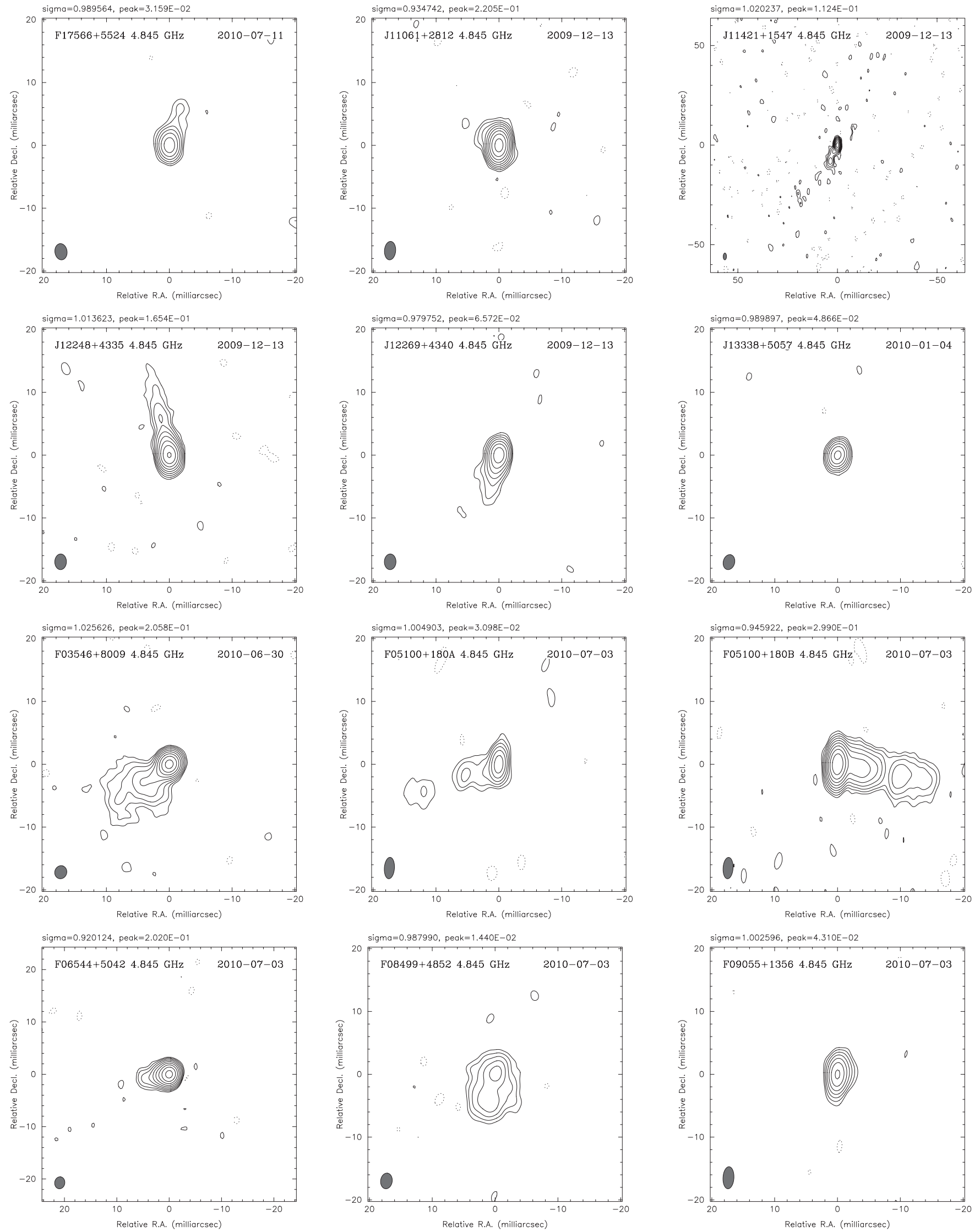

Figure 11. (Continued) 

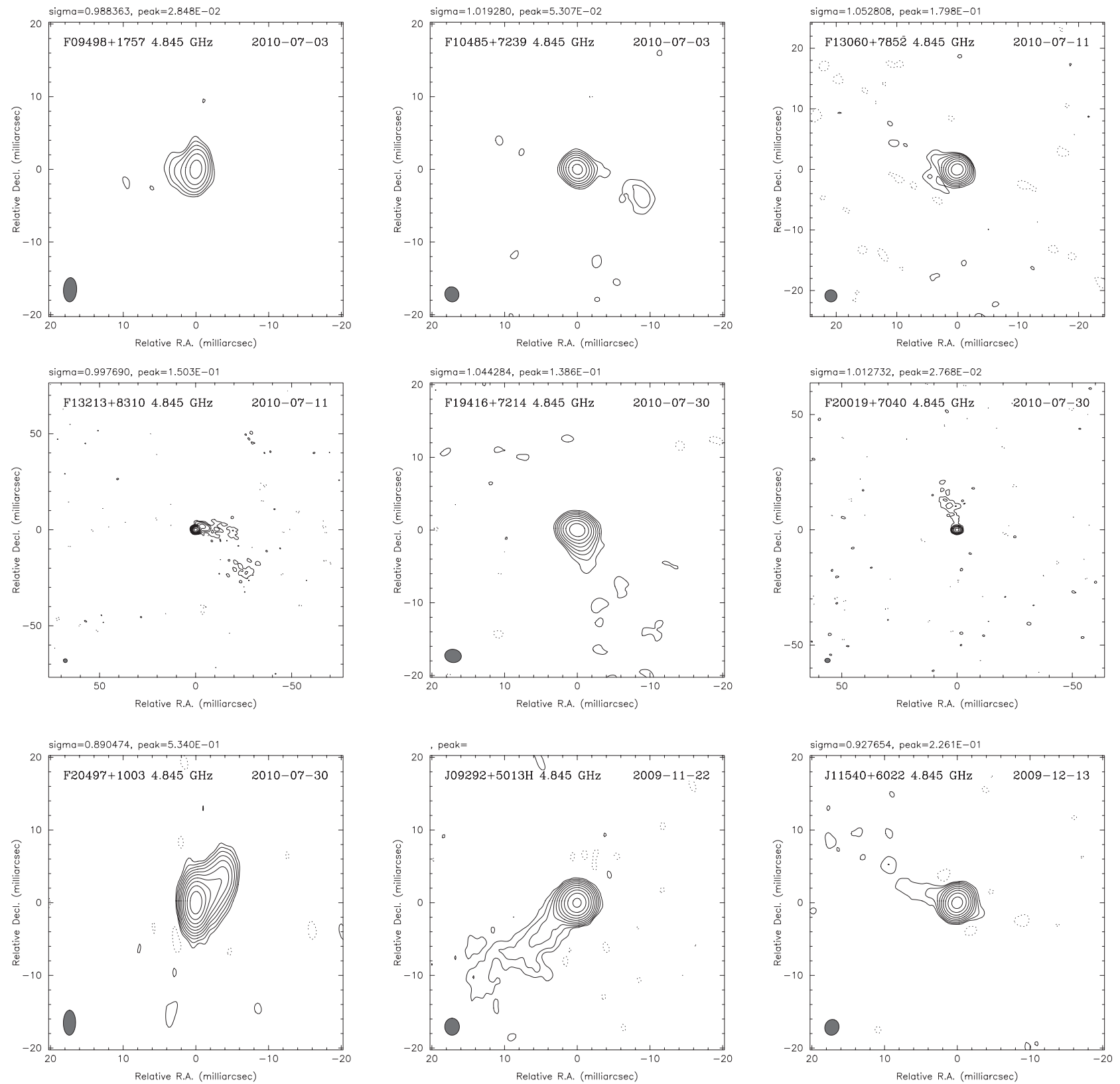

Figure 11. (Continued)

2007). At $5 \mathrm{GHz}$, it is simply a point source. It is also thought to host two intermediate-mass $\left(12,000\right.$ to $\left.43,000 M_{\odot}\right)$ black holes (Feng et al. 2010).

F17250+1151, 1FGL J1725.0+1151, class SJET. This BL Lac object, also known as CRATES J1725+1152, has a redshift of $z=0.018$ (Ciliegi et al. 1993). Its LAT flux was $54.96 \pm 23.25 \times 10^{-9}$ photons $\mathrm{cm}^{-2} \mathrm{~s}^{-1}$. We measured a total VLBA flux density of $63.3 \mathrm{mJy}$. Its core fractional polarization was $3.5 \%$. It is a compact source with a short jet extending about 3.5 mas to the southeast.

\section{2. $A G N$}

The LAT 1FGL contains 28 sources which are classified as "AGNs," which means "other non-blazar AGNs" (Abdo et al. 2010a). We have 12 of these objects in our sample. (Note: 1FGL J0319.7+4130, a.k.a. 3C 84, is discussed above as a low-redshift object.) Some of the objects in this section are radio galaxies, but others may be misidentified FSRQs and BL Lac objects.

J09235+4125, IFGL J0923.2+4121, class LJET. Also known as CRATES J0923+4125, this object had a LAT flux of $40.61 \pm 10.01 \times 10^{-9}$ photons $\mathrm{cm}^{-2} \mathrm{~s}^{-1}$. Its redshift is $z=$ 0.028 (1LAC). This object was observed in two epochs: first in 2006 May and second in 2009 November. In 2006, we found a total VLBA flux density of $165 \mathrm{mJy}$. In 2009, we found a total VLBA flux density of $220 \mathrm{mJy}$. While its core brightness temperature is not unusually high or low for AGN/Other objects, it did change significantly between the two epochs. In 2006, its core brightness temperature was $4.91 \times 10^{9} \mathrm{~K}$. In 2009, we found a core brightness temperature of $2.97 \times 10^{10} \mathrm{~K}$. We detected polarization in the core of this object in both epochs. In 2006 , its core fractional polarization was $5.2 \%$. In 2009, its core fractional polarization was $3.1 \%$. We also detected polarization 
in the jet in 2009 , with a jet fractional polarization of $18.3 \%$. For more discussion on this object, see Linford et al. (2011). It has a jet extending about 9.7 mas to the east.

J12030+6031, 1FGL J1202.9+6032, class LJET. Also known as CRATES J1203+6031, this object had a LAT flux of $44.79 \pm 10.07 \times 10^{-9}$ photons $\mathrm{cm}^{-2} \mathrm{~s}^{-1}$. Its redshift is $z=$ 0.065 (1LAC). This object was observed in two epochs: first in 2006 May and second in 2009 December. Its total VLBA flux density did not change much between the two epochs, but it did show an increase in core brightness temperature. In 2006, its core brightness temperature was $7.22 \times 10^{9}$. In 2009, we found a core brightness temperature of $2.75 \times 10^{10} \mathrm{~K}$. It had strong core polarization in both epochs. In 2006 , we found a core fractional polarization of $9.9 \%$. In 2009 , we found a core fractional polarization of $4.8 \%$. For more discussion on this object, see Linford et al. (2011). It has a long jet extending about 10.5 mas to the south.

J13307+5202, 1FGL J1331.0+5202, class LJET. This object is also called CRATES J1330+5202. Its LAT flux was $41.52 \times 10^{-9}$ photons $\mathrm{cm}^{-2} \mathrm{~s}^{-1}$, but is an upper limit. It has a redshift of 0.688 (1LAC). This object was observed in two epochs: first in 2006 July and second in 2010 January. Its total VLBA flux density and core brightness temperature did not change significantly between the two epochs. We did not detect any polarization from this object in either epoch. For further discussion on this object, see Linford et al. (2011). It has a small jet extending about 7 mas to the southwest.

J16071+1551, 1FGL 1607.1+1552, class LJET. Also called NVSS J160706+155134, its LAT flux was $33.90 \pm$ $7.75 \times 10^{-9}$ photons $\mathrm{cm}^{-2} \mathrm{~s}^{-1}$. Its redshift is $z=0.496$ (1LAC). It is called a BL Lac object in Véron-Cetty \& Véron (2006). This object was observed in two epochs: first in 2006 April and the second in 2010 January. In 2006, we measured a total VLBA flux density of $281 \mathrm{mJy}$. In 2010, we measured a total VLBA flux density of $322 \mathrm{mJy}$. It is a strongly polarized source in both epochs. We measured a core fractional polarization of $3.5 \%$ in 2006 and $4.5 \%$ in 2010 . We also found polarization in the jet in both epochs. In 2006, we measured a jet fractional polarization of $15.5 \%$, and in 2010 we measured $24 \%$. For more information, see Linford et al. (2011). It has a long jet extending about 10 mas to the east.

J16475+4950, 1FGL J1647.4+4948, class LJET. Also called CRATES J1647+4950, this object had a LAT flux of $33.29 \pm$ $8.54 \times 10^{-9}$ photons $\mathrm{cm}^{-2} \mathrm{~s}^{-1}$. It has a redshift of $z=0.047$ (1LAC). This object was observed in two epochs: first in 2006 August and second in 2010 January. Its total VLBA flux density and core brightness temperature did not change significantly between those two epochs. We did not detect any significant core polarization in this object in either epoch, but we did measure $27.1 \%$ jet polarization in 2010 . For more information on this object, see Linford et al. (2011). It has a small jet extending about 7 mas to the southeast.

J17240+4004, 1FGL J1724.0+4002, class LJET. This object is also called CRATES J1724+4004. Its LAT flux was 47.16 $\pm 8.97 \times 10^{-9}$ photons $\mathrm{cm}^{-2} \mathrm{~s}^{-1}$. Its redshift is $z=1.049$ (1LAC). It is tentatively classified as a BL Lac object in VéronCetty \& Véron (2006). This object was observed in two epochs: first in 1998 February and the second in 2010 January. Its total VLBA flux density did not change significantly between the two epochs, but its core brightness temperature more than doubled. In 1998 , its core brightness temperature was $6.08 \times 10^{10}$. In 2010 , we found a core brightness temperature of $3.32 \times 10^{11} \mathrm{~K}$, which is the highest core brightness temperature for the "AGN" objects in our sample. We did not detect any polarization in this object in either epoch. For further discussion on this object, see Linford et al. (2011). It has a long jet extending 9 mas to the northwest.

F02045+1516, IFGL J0204.5+1516, class LJET. This object is also known as $4 \mathrm{C}+15.05$, and it is often classified as an FSRQ (Véron-Cetty \& Véron 2006; Savolainen et al. 2010; Agudo et al. 2010). Its LAT flux was $29.98 \pm 12.42 \times 10^{-9}$ photons $\mathrm{cm}^{-2} \mathrm{~s}^{-1}$. We measured a total VLBA flux density of $1.5 \mathrm{Jy}$. Its redshift is $z=0.405$ (1LAC). Its core brightness temperature was a relatively high $9.52 \times 10^{10} \mathrm{~K}$. We did not detect any polarization from this object. This is one of the sources monitored as part of the MOJAVE program. Savolainen et al. (2010) reported an apparent velocity of $6.3 c$, a Doppler factor of 15.0, and a Lorentz factor of 8.9. It has a bright jet component about 7 mas northwest of the core.

F03250+3403, IFGL J0325.0+3402, class LJET. Also called CRATES J0324+3410, this object is a Seyfert 1 galaxy (Abdo et al. 2009b). It had a LAT flux of $56.29 \pm$ $23.97 \times 10^{-9}$ photons $\mathrm{cm}^{-2} \mathrm{~s}^{-1}$. We measured a total VLBA flux density of $357 \mathrm{mJy}$. It has a redshift of $z=0.061$ (1LAC). We detected polarization in both the core and jet of this object. Its core fractional polarization was $1.0 \%$ and its jet fractional polarization was $33.4 \%$. Zhou et al. (2007) reported the existence of spiral-arm structure in this object based on Hubble Space Telescope images. Antón et al. (2008), using the Nordic Optical Telescope, suggested that the apparent structure could be the result of a merger. In the radio, it has a long straight jet extending about 9.6 mas to the southeast.

F16354+8228, 1FGL J1635.4+8228, class LJET. This object is also known as NGC 6251. It is an unabsorbed Seyfert 2, meaning it has an X-ray hydrogen column density $N_{\mathrm{H}} \leqslant$ $10^{22} \mathrm{~cm}^{-2}$ and does not have a broad-line region (Panessa $\&$ Bassani 2002). It is a well-known radio source with an exceptionally long (about $200 \mathrm{kpc}$ ) and straight jet (Waggett et al. 1977) and is popularly known as the "blowtorch" jet. Its LAT flux was $45.51 \pm 18.77 \times 10^{-9}$ photons $\mathrm{cm}^{-2} \mathrm{~s}^{-1}$. We measured a total VLBA flux density of $637 \mathrm{mJy}$. Its redshift is $z=0.025$ (1LAC). We did not detect any polarization in this object, although it has been reported to be weakly polarized (Chen et al. 2011). It has a long straight jet extending about 8.5 mas to the northwest.

F16410+1143, IFGL J1641.0+1143, LJET. Also called NVSS J164058+114404, this object had a LAT flux of $47.36 \pm$ $20.81 \times 10^{-9}$ photons $\mathrm{cm}^{-2} \mathrm{~s}^{-1}$. We measured a total VLBA flux density of $115 \mathrm{mJy}$. It has a redshift of 0.078 (1LAC). We found a core brightness temperature of $1.65 \times 10^{9} \mathrm{~K}$, which is the lowest of the "AGN'-type objects. We did not detect any polarization for this object. In the CRATES catalog, it is called a flat-spectrum radio source (Healey et al. 2007). It has a jet extending about 10 mas to the west, ending in a semi-detached component.

F17566+5524, 1FGL J1756.6+5524, class LJET. This object is also called CRATES J1757+5523. It had a LAT flux of $27.79 \pm$ $12.00 \times 10^{-9}$ photons $\mathrm{cm}^{-2} \mathrm{~s}^{-1}$. We measured a total VLBA flux density of $47 \mathrm{mJy}$. Its redshift is 0.065 (1LAC). In the CRATES catalog, it is called a flat-spectrum radio source (Healey et al. 2007). We did not detect any polarized flux from this object. It has a jet stretching about 8 mas to the north-northwest.

\subsection{AGN with No Optical ID}

Many sources in the LAT catalog are labeled as "AGU" sources, which means "active galaxy of uncertain type" (Abdo 
et al. 2010a). These sources are associated with radio AGNs, but have no optical identification as yet. We have 16 of them in our sample. It is likely that most of them are FSRQs.

$J 11061+2812,1 F G L J 1106.5+2809$, class PS. This object is also called CRATES J1106+2812. The CRATES catalog lists it as a flat-spectrum radio source (Healey et al. 2007). It had a LAT flux of $34.63 \pm 15.03 \times 10^{-9}$ photons $\mathrm{cm}^{-2} \mathrm{~s}^{-1}$. It has a redshift of 0.847 (Adelman-McCarthy et al. 2008). This object was observed in two epochs: first in 2006 February and the second in 2009 December. In 2006, we found a total VLBA flux density of $276 \mathrm{mJy}$ and in 2009 we measured a total VLBA flux density of $227 \mathrm{mJy}$. Its core brightness temperature more than doubled between the two epochs, going from $5.08 \times 10^{10} \mathrm{~K}$ in 2006 to very high $2.25 \times 10^{11} \mathrm{~K}$ in 2009 . In fact, this object had the highest core brightness temperature of all the "AGU" objects in our sample. We detected polarization in the core of this object in both epochs. In 2006, we measured a core fractional polarization of $1.4 \%$, and in 2009 its core fractional polarization was $4.4 \%$. For more discussion on this object, see Linford et al. (2011). It is a compact, point-source type object.

J11421+1547, IFGLJ1141.8+1549, class LJET. This object is also called CRATES J1142+1547. The CRATES catalog lists it as a flat-spectrum radio source (Healey et al. 2007). It had a LAT flux of $12.87 \pm 5.19 \times 10^{-9}$ photons $\mathrm{cm}^{-2} \mathrm{~s}^{-1}$. There is no published redshift for this object. This object was observed in two epochs: first in 2006 February and second in 2009 December. Its total VLBA flux density was $172 \mathrm{mJy}$ in 2006 and $139 \mathrm{mJy}$ in 2009. Its core brightness temperature increased between the two epochs from $5.62 \times 10^{10} \mathrm{~K}$ to 9.15 $\times 10^{10} \mathrm{~K}$, which is high for an AGN/Other type object. We detected polarization in the core of this object in both epochs. In 2006, the core fractional polarization was $6.3 \%$ and in 2009 the core fractional polarization was $5.5 \%$. We also detected polarization in the jet in 2009. The jet fractional polarization was $31.1 \%$. For more information on this object, see Linford et al. (2011). It has a jet extending about 11.5 mas to the southeast.

J12248+4335 (class LJET) \& J12269+4340 (class SJET), IFGL J1225.8+4336. The LAT source 1FGL J1225.8+4336, with a LAT flux of $36.15 \pm 15.51 \times 10^{-9}$ photons $\mathrm{cm}^{-2} \mathrm{~s}^{-1}$, is associated with two VIPS sources, both of which were observed in two epochs: first in 2006 May and second in 2009 December. First, J12248+4335 (probability: 94\%, 1LAC), also called NVSS J122451+433520. This object is called a BL Lac object by Plotkin et al. (2008) but listed as an "Unknown" type in both 1FGL and 1LAC. It has a redshift of $z=1.07491$ (Sowards-Emmerd et al. 2003). In 2006, we measured a total VLBA flux density of 174 mJy and in 2009 we measured a total VLBA flux density of $207 \mathrm{mJy}$. Its core brightness temperature increased from $1.80 \times 10^{10} \mathrm{~K}$ in 2006 to $3.15 \times 10^{10} \mathrm{~K}$ in 2009. We also detected polarization in the core and jet of this object in both epochs. In 2006, its core fractional polarization was $7 \%$ and its jet fractional polarization was $18 \%$. In 2009 , its core fractional polarization was $5.4 \%$ and its jet fractional polarization was $42.6 \%$. It has a jet extending about 8 mas to the northeast. Second, J12269+4340 (probability: 87\%, 1LAC), or CRATES J1226+4340, is an FSRQ (Véron-Cetty \& Véron 2006 and 1LAC). It has a redshift of $z=2.002$ (1LAC). Its total VLBA flux density and core brightness temperature did not change significantly between the two epochs. We detected core polarization only in the 2009 observation. We found core fractional polarization of $4.2 \%$. It is a compact source with a possible jet extending about 5.6 mas to the south.
J13338+5057, IFGL J1333.2+5056, class PS. This object, also called CLASS J1333+5057, has no published classification. Its LAT flux was $64.33 \pm 27.63 \times 10^{-9}$ photons $\mathrm{cm}^{-2} \mathrm{~s}^{-1}$. It has a redshift of $z=1.362$ (Abdo et al. 2009). It was observed in two epochs: first in 2006 August and the second in 2010 January. It was very dim in 2006, with a total VLBA flux density of $40 \mathrm{mJy}$, but it doubled to $80.2 \mathrm{mJy}$ in 2010 . Its core brightness temperature also increased by about a factor of two from $6.24 \times$ $10^{9} \mathrm{~K}$ in 2006 to $1.46 \times 10^{10} \mathrm{~K}$ in 2010 . We did not detect any polarization in this object in either epoch. It is a compact, point-source type object.

F03546+8009, 1FGL J0354.6+8009, class LJET. This object is also known as CRATES J0354+8009. The CRATES catalog lists it as a flat-spectrum radio source (Healey et al. 2007). Its LAT flux was $46.05 \pm 8.82 \times 10^{-9}$ photons $\mathrm{cm}^{-2} \mathrm{~s}^{-1}$. We measured a total VLBA flux density of $280 \mathrm{mJy}$. There is no published redshift for this object. It was a very strongly polarized source with a core fractional polarization of $14.2 \%$. It has a broad, diffuse jet extending about 9.8 mas to the southeast. It also had a large opening angle of $35^{\circ}$. Britzen et al. (2008) measured the kinematics of two of the jet components for this object and found apparent velocities of $0.007 c$ for the one nearer the core and $0.064 c$ for the one further from the core.

F05100+180A\&B (both class LJET), 1FGL J0510.0+1800. The LAT source 1FGL J0510.0+1800, with a LAT flux of $33.38 \pm 12.39 \times 10^{-9}$ photons $\mathrm{cm}^{-2} \mathrm{~s}^{-1}$, is associated with two of our radio sources. The first, F05100+180A, is also known as CRATES J0509+1806. In 1LAC, its optical type is listed as "Unknown." The CRATES catalog lists it as a flatspectrum radio source (Healey et al. 2007). It has no published redshift. We measured a total VLBA flux density of $44.8 \mathrm{mJy}$. We did not detect any polarized flux from this object. It has a long jet extending to the east with a detached component about 16 mas from the center of the core. The second radio source, F05100+180B, is an FSRQ also known as PKS 0446+17. We measured a total VLBA flux density of $441 \mathrm{mJy}$. We detected polarized flux in both the core and jet of this object. Its core fractional polarization was $3.8 \%$ and its jet fractional polarization was $12.8 \%$. It has a long jet extending about 13 mas to the west.

F06544+5042, 1FGL J0654.4+5042, class SJET. This object is also called CRATES J0654+5042. The CRATES catalog lists it as a flat-spectrum radio source (Healey et al. 2007). Its LAT flux was $42.02 \pm 7.63 \times 10^{-9}$ photons $\mathrm{cm}^{-2} \mathrm{~s}^{-1}$. Abdo et al. (2010c) report that its $\gamma$-ray flux $(E>300 \mathrm{MeV})$ is variable. We measured a total VLBA flux density of $240 \mathrm{mJy}$. It has no published redshift. We found a core fractional polarization of $8.1 \%$. It has a small jet extending about 4.7 mas to the east.

F08499+4852, 1FGL J0849.9+4852, class LJET. This object is also known as CRATES J0850+4854. The CRATES catalog lists it as a flat-spectrum radio source (Healey et al. 2007). Its LAT flux was $29.87 \pm 6.99 \times 10^{-9}$ photons $\mathrm{cm}^{-2} \mathrm{~s}^{-1}$. We measured a total VLBA flux density of $60.1 \mathrm{mJy}$. There is no published redshift for this object. We found a core brightness temperature of $1.20 \times 10^{9} \mathrm{~K}$, which is the lowest of all the AGN/Other type objects. We did not detect any polarization in this object. It has a small jet extending about 8 mas to the south. It also has a large opening angle of 54.2 .

F09055+1356, IFGL J0905.5+1356, class SJET. This object is also called CRATES J0905+1358. The CRATES catalog lists it as a flat-spectrum radio source (Healey et al. 2007). Its LAT flux was $18.39 \pm 7.21$ photons $\mathrm{cm}^{-2} \mathrm{~s}^{-1}$. We measured a total 
VLBA flux density of $48.5 \mathrm{mJy}$. It has no published redshift. We did not detect any polarization in this object. It is a compact object with a very short jet extending about 1.3 mas to the west.

F09498+1757, 1FGL J0949.8+1757, class SJET. This object is also known as CRATES J0950+1804. The CRATES catalog lists it as a flat-spectrum radio source (Healey et al. 2007). It had an upper limit on its LAT flux of $18.85 \times 10^{-9}$ photons $\mathrm{cm}^{-2} \mathrm{~s}^{-1}$. We measured a total VLBA flux density of $37.2 \mathrm{mJy}$. Its redshift is 0.69327 (Adelman-McCarthy et al. 2008). We did not detect any polarized flux from this object. It appears to have a short jet extending about 5 mas to the east.

F10485+7239, 1FGL J1048.5+7239, class LJET. This object is also known as CRATES J1047+7238. The CRATES catalog lists it as a flat-spectrum radio source (Healey et al. 2007). Its LAT flux was $44.18 \pm 19.09 \times 10^{-9}$ photons $\mathrm{cm}^{-2} \mathrm{~s}^{-1}$. We measured a total VLBA flux density of $61 \mathrm{mJy}$. It has no published redshift. We found a core fractional polarization of $5.2 \%$. It has a jet extending about 12 mas to the west.

F13060+7852, 1FGL J1306.0+7852, class SJET. This object is also called CRATES J1305+7854. The CRATES catalog lists it as a flat-spectrum radio source (Healey et al. 2007). Its LAT flux was $17.53 \pm 6.82 \times 10^{-9}$ photons $\mathrm{cm}^{-2} \mathrm{~s}^{-1}$. We measured a total VLBA flux density of $213 \mathrm{mJy}$. There is no published redshift for this object. We found a core fractional polarization of $4.2 \%$. It has a small jet extending about 5 mas to the northeast.

F13213+8310, 1FGL J1321.3+8310, class LJET. This object is also called CRATES J1321+8316. The CRATES catalog lists it as a flat-spectrum radio source (Healey et al. 2007). Its LAT flux was $27.88 \pm 11.62 \times 10^{-9}$ photons $\mathrm{cm}^{-2} \mathrm{~s}^{-1}$. We measured a total VLBA flux density of $232 \mathrm{mJy}$. It has a redshift of $z=$ 1.024 (Britzen et al. 2007). We did not detect any polarization in this object. It has a long, diffuse jet extending about 22.5 mas to the west. It also appears to have an older, detached jet component about 33 mas to the southwest. Britzen et al. (2008) had measurements for the kinematics on two components in this source's jet, but for some reason they did not use the redshift quoted earlier. Using that redshift, and Britzen's total proper

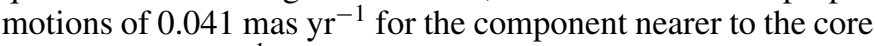

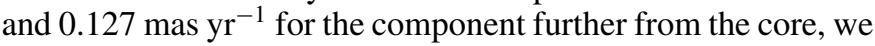
find apparent velocities of $1.08 c$ and $3.35 c$, respectively.

F19416+7214, 1FGL J1941.6+7214, class SJET. This object is also called CRATES J1941+7221. There is no published classification for it. Its LAT flux was $66.46 \pm$ $29.70 \times 10^{-9}$ photons $\mathrm{cm}^{-2} \mathrm{~s}^{-1}$. We measured a total VLBA flux density of $775 \mathrm{mJy}$. There is no published redshift for this object. We did not detect any polarization in this object. It has a small jet extending about 4 mas to the south.

F20019+7040, 1FGL J2001.9+7040, class LJET. This object is also known as CRATES J2001+7040. The CRATES catalog lists it as a flat-spectrum radio source (Healey et al. 2007). Its LAT flux was $27.18 \pm 10.17 \times 10^{-9}$ photons $\mathrm{cm}^{-2} \mathrm{~s}^{-1}$. We measured a total VLBA flux density of $36.5 \mathrm{mJy}$. There is no published redshift for this object. We did not detect any polarization in this object. It has a long, diffuse jet extending about 14 mas to the north.

F20497+1003, IFGL J2049.7+1003, class LJET. This object is also known as CRATES J2049+1003. It may also be the EGRET source 3EG J2046+0933 (Bloom 2008). The CRATES catalog lists it as a flat-spectrum radio source (Healey et al. 2007). Its LAT flux was $58.69 \pm 25.99 \times 10^{-9}$ photons $\mathrm{cm}^{-2} \mathrm{~s}^{-1}$. We measured a total VLBA flux density of $698 \mathrm{mJy}$. It has no published redshift. We found a core brightness temperature of $6.07 \times 10^{10} \mathrm{~K}$. We found a very low core fractional polarization of $0.8 \%$. It has a jet extending about 6.3 mas to the northwest.

\subsection{Unidentified Sources}

Our sample contains two objects for which no optical classification is given in the 1FGL catalog.

J09292+5013, IFGL J0929.4+5000, class LJET. In our previous paper (Linford et al. 2011), we identified this source as a BL Lac object. It is associated with the BL Lac object VIPS J09292+5013 (CRATES J0929+5013), but with a low probability $(67 \%, 1 \mathrm{LAC})$. For this paper, we treated it as a BL Lac object. It had a LAT flux of $21.40 \pm 9.29 \times 10^{-9}$ photons $\mathrm{cm}^{-2} \mathrm{~s}^{-1}$. The two epochs for which we have observations of this object are 1998 February and 2009 November. It has a redshift of $z=$ 0.370387 (Abazajian et al. 2005). Its total VLBA flux density did not change significantly between the two epochs. In 1998, we found a core brightness temperature of $6.67 \times 10^{10} \mathrm{~K}$ and in 2009 we found a core brightness temperature of $4.81 \times 10^{10} \mathrm{~K}$. Even with this somewhat reduced core brightness temperature, it was still in the upper $33 \%$ of BL Lac objects in the current sample. It was a strongly polarized source with a core fractional polarization of $16.9 \%$.

$J 11540+6022,1 F G L J 1152.1+6027$, class PS. This is associated with VIPS J11540+6022 (CRATES J1154+6022), a source of unknown type, with a probability of $73 \%$ (1LAC). Healey et al. (2007) call it a flat-spectrum radio source. Its LAT flux was $46.21 \pm 20.76 \times 10^{-9}$ photons $\mathrm{cm}^{-2} \mathrm{~s}^{-1}$. We measured a total VLBA flux density of $232 \mathrm{mJy}$. It has no published redshift. We found a core brightness temperature of $3.93 \times 10^{11} \mathrm{~K}$, which is the highest core brightness temperature of all the "Other" type objects and the 16th highest in our entire sample. We found a core fractional polarization of $1.4 \%$.

\section{DISCUSSION AND CONCLUSIONS}

\subsection{Polarization and Magnetic Fields}

The widely accepted picture of AGN central engine is a spinning supermassive black hole surrounded by an accretion disk (Blandford 1976; Lovelace 1976; Urry \& Padovani 1995). In order to launch and collimate the jets, most models include a strong magnetic field which is coiled into a helical shape by the rotation of the accretion disk or the black hole. Meier (2005) proposed a model where the magnetic field lines originate in the accretion disk and then thread through the ergosphere (the region near a rotating black hole where spacetime itself is rotating as a result of frame dragging) of the rotating central black hole. This allows for the black hole to tightly wind the magnetic field lines, leading to reconnection events which can launch fastmoving material. This may be the source of the $\gamma$-ray-emitting regions in our blazars.

We know that strong, uniform magnetic fields lead to polarized emission. Because the majority (76\%) of our $\gamma$-ray bright sources showed significant polarization in their cores, it is obvious that they have strong, well-ordered magnetic fields in their centers. The fact that the non-LAT sources are polarized less often (see Figure 12) leads us to believe that the strong, uniform magnetic fields are somehow tied to the $\gamma$-ray emission. Also, recall from Section 5.3 that the core fractional polarization appeared to increase during LAT detection. Furthermore, Abdo et al. (2010d) noted a dramatic change in the optical polarization orientation angle coincided with strong $\gamma$-ray emission in 


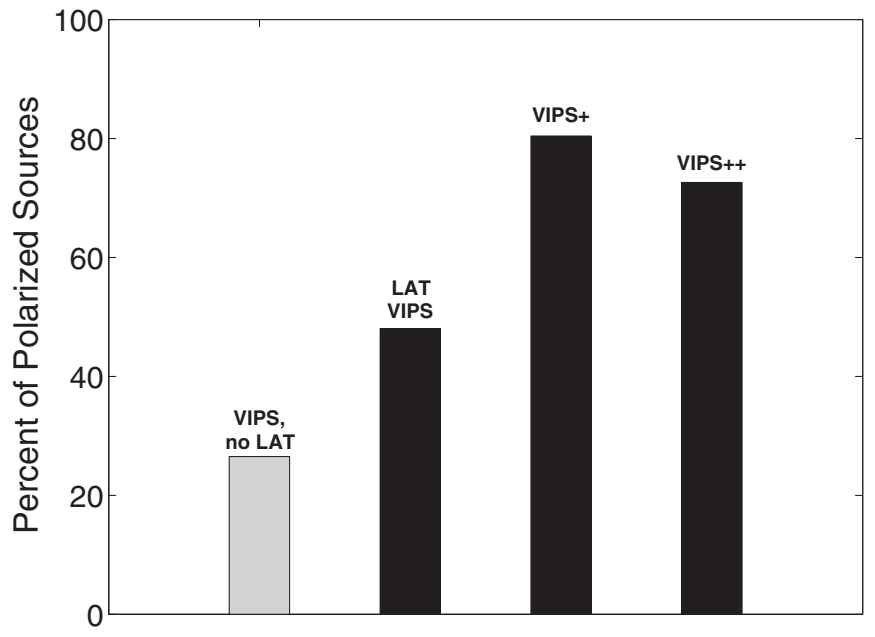

Figure 12. Percentage of sources found to be polarized in various VLBI samples including VIPS (observations made prior to or during 2006), VIPS+ (second epoch VIPS observations made contemporaneously with Fermi observations, 2009-2010), and VIPS++ (contemporaneous observations of additional LAT blazars, 2010). Gray indicates the sample with non-LAT-detected sources and black indicates LAT samples.

3C279. A possible explanation for this is that the magnetic fields in the cores become stronger (field lines wound more tightly) and more uniform (lines pulled into a less chaotic configuration) when $\gamma$-ray emission occurs. In other words, following from the model in Meier (2005) discussed above, the spinning black hole winds up the magnetic field lines until there is a reconnection event which launches a new, fast-moving jet component. This new component upscatters background photons to $\mathrm{GeV}$ energies via inverse Compton processes (e.g., Björnsson 2010; Tavecchio et al. 2011; Abdo et al. 2011). Meier (2005) expected several reconnection events in succession, followed by a time when the field has pulled back from the black hole. Thus, the objects for which we do not detect polarization in the core could be in the state where the field is not being wound up by the black hole.

\subsection{Opening Angles}

While we did not have a large sample of objects with measured opening angles, we did find a hint that the LAT and non-LAT distributions are different. The K-S test gave a $0.4 \%$ probability that the LAT and non-LAT distributions of BL Lac object and FSRQ opening angles are taken from the same parent sample. This is not a highly significant result, but it is tantalizing in view of what other studies have reported (e.g., Pushkarev et al. 2009; Ojha et al. 2010b; Lister et al. 2011).

\subsection{BL Lac Objects}

The only significant difference between the LAT and nonLAT BL Lac objects was that the LAT BL Lac objects are polarized more often. However, 10 of the 24 non-LAT BL Lac objects showed significant core polarization, so core polarization itself is not enough to separate the two populations. It seems likely, therefore, that all BL Lac objects produce $\gamma$-rays, but we simply do not detect all of them with the LAT. It is well known that BL Lac objects are highly variable sources in radio and $\gamma$-ray bands (Abdo et al. 2011). It is possible that LAT does not detect some BL Lac objects because they have lower than average Doppler factors, either as a result of lower velocities and/or jet orientations further from the line-of-sight. Lister et al. (2009c) measured the kinematics of both LAT and non-LAT BL Lac objects, and found two non-LAT BL Lac objects with maximum jet material speeds higher than the LAT BL Lac objects. However, they only had 21 BL Lac objects, 10 of which were LAT detected. Further study of BL Lac object kinematics is needed before we can claim LAT BL Lac objects tend to have higher bulk material velocities. It is also possible that BL Lac objects occasionally enter a state where $\gamma$-ray production ceases or is at least significantly reduced. This question may be answered with continued monitoring of BL Lac objects with LAT and future instruments.

\subsection{FSRQs}

The LAT FSRQs exhibited several significant differences from the non-LAT FSRQs. The LAT FSRQs had higher radio flux densities, higher core brightness temperatures, and were polarized much more often than the non-LAT FSRQs. The LAT FSRQs also seemed to have higher jet brightness temperatures, although this is a marginal result. It is also possible that the LAT FSRQs have larger opening angles than non-LAT FSRQs, but this result is tentative and is not strongly supported by the K-S test.

As with the BL Lac objects, we saw significant variation in flux density, core brightness temperature, and core polarization for those sources for which we had two epochs of observations. However, as a group the FSRQs did not change significantly in flux density or core brightness temperature between the two epochs. We did find an increase in the number of sources with strong core polarization.

About 90\% (96 of 107) of the LAT FSRQs showed significant polarization in their cores. Compare this with only about $33 \%$ of the non-LAT FSRQs showing core polarization, and we can see that core polarization is a strong indicator of $\gamma$-ray emission. Of the 44FSRQs for which we had observations in two epochs, 28 showed higher core fractional polarization during LAT detection. Only 12 showed a decrease in core fractional polarization. So, it appears that the cores of FSRQs tend to become more polarized during LAT detection.

\subsection{Other Non-blazar AGNs}

We found two significant differences between the LAT and non-LAT non-blazar AGNs. The first is the total radio flux density. The LAT non-blazar AGNs tended to have higher flux density than their non-LAT counterparts. The K-S test resulted in a $8.8 \times 10^{-4}$ chance that the LAT and non-LAT distributions are drawn from the same parent distribution. Second, the LAT nonblazar AGNs are polarized much more frequently. We detected polarization in about 43\% (13 of 30) of our LAT sources, compared to only about $20 \%$ (102 of 515) for our non-LAT sample. As with the BL Lac objects and FSRQs, the non-blazar AGNs are not necessarily more strongly polarized, but polarized more often.

Also, there remains a definite lack of CSO candidates among LAT-detected AGNs, despite some predictions to the contrary (e.g., Stawarz et al. 2008).

We thank the anonymous referee for helpful comments on the manuscript. We thank Steve Tremblay, Marcello Giroletti and Bob Hastman for their helpful advice and comments. We also thank Steve Myers and Josh Marvil for their help with obtaining EVLA observations of several sources for polarization angle calibration. The National Radio Astronomy Observatory is a facility of the National Science Foundation operated under 
cooperative agreement by Associated Universities, Inc. This work made use of the Swinburne University of Technology software correlator, developed as part of the Australian Major National Research Facilities Programme and operated under license. The NASA/IPAC Extragalactic Database (NED) is operated by the Jet Propulsion Laboratory, California Institute of Technology, under contract with the National Aeronautics and Space Administration. We thank NASA for support under FERMI grant GSFC 21078/FERMI08-0051 and the NRAO for support under Student Observing Support Award GSSP10-011.

\section{REFERENCES}

Abazajian, K., Adelman-McCarthy, J. K., Agüeros, M. A., et al. 2005, AJ, 129. 1755

Abdo, A. A., Ackermann, M., Ajello, M., et al. 2009, ApJ, 700, 597

Abdo, A. A., Ackermann, M., Ajello, M., et al. 2009b, ApJ, 707, L142

Abdo, A. A., Ackermann, M., Ajello, M., et al. 2010a, ApJS, 188, 405

Abdo, A. A., Ackermann, M., Ajello, M., et al. 2010b, ApJ, 715, 429

Abdo, A. A., Ackermann, M., Ajello, M., et al. 2010c, ApJ, 722, 520

Abdo, A. A., Ackermann, M., Ajello, M., et al. 2010d, Nature, 463, 919

Abdo, A. A., Ackermann, M., Ajello, M., et al. 2011, ApJ, 730, 101

Adelman-McCarthy, J. K., Agüeros, M. A., Allam, S. S., et al. 2008, ApJS, 175 297

Agudo, I., Thum, C., Wiesenmeyer, H., \& Krichbaum, T. P. 2010, ApJS, 189, 1

Antón, S., Browne, I. W. A., \& Marchã, M. J. 2008, A\&A, 490, 583

Atwood, B. W., Abdo, A. A., Ackermann, M., et al. 2009, ApJ, 697, 1071

Björnsson, C. I. 2010, ApJ, 723, 417

Blandford, R. D. 1976, MNRAS, 176, 465

Bloom, S. D. 2008, AJ, 136, 1533

Britzen, S., Brinkmann, W., Campbell, R. M., et al. 2007, A\&A, 476, 759

Britzen, S., Vermeulen, R. C., Campbell, R. M., et al. 2008, A\&A, 484, 119

Chen, R., Peng, B., Strom, R. G., \& Wei, J. 2011, MNRAS, 412, 2433

Ciliegi, P., Bassani, L., \& Caroli, E. 1993, ApJS, 85, 111

Deller, A. T., Brisken, W. F., Phillips, C. J., et al. 2011, PASP, 123, 275

de Vaucouleurs, G., de Vaucouleurs, A., Corwin, H. G., et al. 1991, Third

Reference Catalogue of Bright Galaxies (RC3) (New York: Springer)

Feng, H., Rao, F., \& Kaaret, P. 2010, ApJ, 710, L137

Giroletti, M., Reimer, A., Fuhrmann, L., Pavlidou, V., \& Richards, J. L. 2010, 2009 Fermi Symposium, eConf Proceedings C091122, arXiv:1001.5123

Greisen, E. W. 2003, Astrophys. Space Sci. Libr., 285, 109

Healey, S. E., Romani, R. W., Cotter, G., et al. 2008, ApJS, 175, 97

Healey, S. E., Romani, R. W., Taylor, G. B., et al. 2007, ApJS, 171, 61

Helmboldt, J. F., Taylor, G. B., Tremblay, S., et al. 2007, ApJ, 658, 203

Helmboldt, J. F., Taylor, G. B., Walker, R. C., \& Blandford, R. D. 2008, ApJ, 681,897
Hinshaw, G., Weiland, J. L., Hill, R. S., et al. 2009, ApJS, 180, 225

Homan, D. C., Kadler, M., Kellermann, K. I., et al. 2009, ApJ, 706, 1253

Huber, P. J., \& Ronchetti, E. M. 2009, Robust Statistics (2nd ed.; Hoboken, NJ: Wiley)

Jorstad, S. G., Marscher, A. P., Larionov, V. M., et al. 2010, ApJ, 715, 362

Kovalev, Y. Y., Aller, H. D., Aller, M. F., et al. 2009, ApJ, 696, L17

Kovalev, Y. Y., Kellermann, K. I., Lister, M. L., et al. 2005, AJ, 130, 2473

Linford, J. D., Taylor, G. B., Romani, R. W., et al. 2011, ApJ, 726, 16

Lister, M. L., Aller, H. D., Aller, M. F., et al. 2009a, AJ, 137, 3718

Lister, M. L., Aller, M., Aller, H., et al. 2011, ApJ, 742, 27

Lister, M. L., Cohen, M. H., Homan, D. C., et al. 2009b, AJ, 138, 1874

Lister, M. L., Homan, D. C., Kadler, M., et al. 2009c, ApJ, 696, L22

Lovelace, R. 1976, Nature, 262, 649

Marscher, A. P. 2006, in AIP Conf. Proc. 856, Relativistic Jets: The Common Physics of AGN, Microquasars, and Gamma-Ray Bursts, ed. P. A. Hughes \& J. N. Bregman (Melville, NY: AIP), 1

Marscher, A. P., Jorstad, S. G., Larionov, V. M., et al. 2010, ApJ, 710, L126

Meier, D. L. 2005, Ap\&SS, 300, 55

Mutchler, M., Bond, H. E., Christian, C. A., et al. 2007, PASP, 119, 1

Ojha, R., Kadler, M., Böck, M., et al. 2010a, A\&A, 519, A45

Ojha, R., Kadler, M., Böck, M., et al. 2010b, in 2009 Fermi Symposium, C091122

Panessa, F., \& Bassani, L. 2002, A\&A, 394, 435

Plotkin, R. M., Anderson, S. F., Hall, P. B., et al. 2008, AJ, 135, 2453

Press, W. H., Flannery, B. P., Teukolksy, S. A., \& Vetterin, W. T. 1986, Numerical Recipes: The Art of Scientific Computing (Cambridge: Cambridge Univ. Press)

Pushkarev, A. B., Kovalev, Y. Y., Lister, M. L., \& Savolainen, T. 2009, A\&A, 507, L33

Savolainen, T., Homan, D. C., Hovatta, T., et al. 2010, A\&A, 512, A24

Schinzel, F. K., Lobanov, A. P., Jorstad, S. G., et al. 2010, in Fermi Meets Jansky: AGN in Radio and Gamma-Rays, ed. T. Savolainen, E. Ros, R. W. Porcas, \& J. A. Zensus (Bonn: MPIfR), 175

Shepherd, M. C. 1997, in ASP Conf. Ser. 125, Astronomical Data Analysis Software and Systems VI, ed. G. Hunt \& H. E. Payne (San Francisco, CA: ASP), 77

Sowards-Emmerd, D., Romani, R. W., \& Michelson, P. F. 2003, ApJ, 590, 109 Stawarz, L., Ostorero, L., Begelman, M. C., et al. 2008, ApJ, 680, 911

Tavecchio, F., Becera-Gonzales, J., Ghisellini, G., et al. 2011, A\&A, 534, 86

Taylor, G. B., Fassnacht, C. D., Sjouwerman, L. O., et al. 2005, ApJS, 159, 27

Taylor, G. B., Gugliucci, N. E., Fabian, A. C., et al. 2006, MNRAS, 368, 1500

Taylor, G. B., Healey, S. E., Helmboldt, J. F., et al. 2007, ApJ, 671, 1355

Urry, C. M., \& Padovani, P. 1995, PASP, 107, 803

Véron-Cetty, M. P., \& Véron, P. 2006, A\&A, 455, 773

Waggett, P. C., Warner, P. J., \& Baldwin, J. E. 1977, MNRAS, 181, 465

Walker, R. C., Dhawan, V., Romney, J. D., Kellermann, K. I., \& Vermeulen, R. C. 2000, ApJ, 530, 233

Zhou, H. Y., Wang, T., Yuan, W., et al. 2007, ApJ, 658, L13 